* Fermilab

Steering

Group

Report 
Fermilab Director Pier Oddone convened the Fermilab Steering Group in March 2007. Members comprised particle and accelerator scientists from Fermilab and the national community. Fermilab Deputy Director Young-Kee Kim served as chair. The Steering Group subsequently formed subgroups to provide advice on the best physics opportunities that new facilities could offer. These subgroups drew upon university and laboratory scientists largely from outside Fermilab.

The Steering Group took a number of steps to obtain as much input as possible from a broad spectrum of the U.S. particle- and accelerator-physics community. The Steering Group chair gave presentations and conducted town-hall-style sessions at meetings of all the major collaborations at Fermilab (CDF, DZero, MINOS, MINERvA, MiniBooNE, SciBooNE, NOvA), at US CMS and International Linear Collider Tesla Technology Collaboration meetings, at the June 6-7 Annual Users Meeting of Fermilab, at the June 7 Users Meeting at Stanford Linear Accelerator Center and at major laboratory seminars (Argonne National Laboratory, Brookhaven National Laboratory, and Lawrence Berkeley National Laboratory). The chair also communicated to the U.S. particle physics and accelerator physics community through the Division of Particles and Fields and the Division of Physics of Beams of the American Physical Society. These sessions advised the community of the Steering Group's purpose, the process it would follow and the mechanism by which it planned to advise the Fermilab director; and how it would provide input to the Particle Physics Project Prioritization Panel, the High Energy Physics Advisory Panel, and the funding agencies. In addition, the Steering Group solicited input on physics possibilities from the entire community either in the form of letters or in one-page expressions of interest. In its few months of existence, the Steering Group received 17 expressions of interest and seven letters. Input from the community demonstrated a broad interest in a domestic facility that supports a strong U.S. accelerator-based program of particle physics.

The Steering Group held open meetings throughout the process of its deliberations, inviting the chairs of the Fermilab and SLAC users' organizations, the chairs of HEPAP and P5, leaders of the Global Design Effort for the ILC and representatives of funding agencies to attend and contribute.

Fermilab has a long history of community input into its physics program and in years past has held many summer studies to consider the best options for new accelerators being developed at the laboratory. Given the short timeline for the Steering Group report, such a summer study was not possible. However, Fermilab intends to conduct such a study should a decision go forward to provide R\&D support for Project $X$ at the intensity frontier. The full span of physics made possible by the proposed high-intensity proton source will be the subject of a future formal call for proposals. However, the Fermilab Physics Advisory Committee and advisory panels including P5 and HEPAP, convened by the funding agencies, will elucidate the prioritization of such experiments. 


\section{Steering Group}

Eugene Beier

University of Pennsylvania

Joel Butler

Fermi National Accelerator Laboratory

Sally Dawson

Brookhaven National Laboratory

Helen Edwards

Fermi National Accelerator Laboratory

Thomas Himel

Stanford Linear Accelerator Center

Stephen Holmes

Fermi National Accelerator Laboratory

Young-Kee Kim, Chair

Fermi National Accelerator Laboratory/ University of Chicago

\section{Contributors}

Neutrino Physics Group

Eugene Beier

University of Pennsylvania

Deborah Harris

Fermi National Accelerator Laboratory

Edward Kearns

Boston University

Boris Kayser

Fermi National Accelerator Laboratory

Sacha Kopp

The University of Texas at Austin

Andrew Lankford, Chair

University of California, Irvine

William Louis

Los Alamos National Laboratory

Andrew Lankford

University of California, Irvine

David McGinnis

Fermi National Accelerator Laboratory

Sergei Nagaitsev

Fermi National Accelerator Laboratory

Tor Raubenheimer

Stanford Linear Accelerator Center

Vladimir Shiltsev

Fermi National Accelerator Laboratory

Maury Tigner

Cornell University

Hendrik Weerts

Argonne National Laboratory

Precision Physics Group

Joel Butler

Fermi National Accelerator Laboratory

Brendan Casey

Fermi National Accelerator Laboratory/ Brown University

Sally Dawson, Chair

Brookhaven National Laboratory

Christopher Hill

Fermi National Accelerator Laboratory

Daniel Kaplan

Illinois Institute of Technology

Yury Kolomensky

Lawrence Berkeley National Laboratory/

University of California, Berkeley

William Molzon

University of California, Irvine

Kevin Pitts

University of Illinois at Urbana-Champaign

Frank Porter

California Institute of Technology

Robert Tschirhart

Fermi National Accelerator Laboratory

Hendrik Weerts

Argonne National Laboratory
Production Editors

Judith Jackson

Fermi National Accelerator Laboratory

Joseph Lykken

Fermi National Accelerator Laboratory

Design and Production

Sandbox Studio

Chicago, Illinois 
Fermilab, the nation's primary laboratory for particle physics, proposes a plan to maintain leadership for the laboratory and U.S. particle physics in the quest to discover the fundamental nature of the physical universe in the decades ahead.

Discoveries of the physics of the Quantum Universe will come from powerful nextgeneration particle accelerators. Fermilab's Tevatron, currently the world's most powerful particle accelerator, will shut down by the end of this decade after the Large Hadron Collider at CERN begins operations. At the LHC, U.S. physicists will join scientists from around the world in the exploration of the physics of the Terascale. To follow the LHC, physicists have proposed the International Linear Collider as the highest priority. Globally funded and operated, the ILC would build on LHC results and illuminate Terascale science. Fermilab will work to host the proposed ILC in the U.S. as soon as possible, maintaining the nation's historic leadership of frontier particle physics.

Should events postpone the start of the ILC, Fermilab has developed a plan to keep the laboratory and particle physics in the U.S. on the pathway to discovery. Using ILC technology, Fermilab would build an intensity-frontier accelerator at about one percent of the ILC's length and combine it with existing Fermilab accelerators to create Project X. Project X's intense proton beams would give Fermilab's scientific users a new way into the world of neutrinos and precision physics, where physicists expect to discover answers to compelling Quantum Universe questions. With its ILC technology, Project X would spur U.S industrialization and reduce costs of ILC components while advancing accelerator science for future applications in particle physics and beyond.

Fermilab's plan would maintain the nation's leadership in particle physics, keeping the laboratory and U.S. particle physics on the pathway to discovery both at the Terascale, with the LHC and the ILC, and in the domain of neutrinos and precision physics at the intensity frontier. 


\section{Contents}

4 Chapter 1

Executive Summary: A Plan for Fermilab

8 Chapter 2

Fermilab and the Quantum Universe

12 Chapter 3

Fermilab and the ILC

14 Chapter 4

Physics at the Intensity Frontier

20 Chapter 5

Facilities for the Intensity Frontier

26 Chapter 6

Beyond the ILC and the LHC

28 Chapter 7

A Fermilab Plan for Discovery

32 Appendix 


\section{Chapter 1 Executive Summary: A Plan for Fermilab}



particle physics on the pathway to discovery, both at the Terascale with the LHC and the ILC and in the domain of neutrinos and precision physics with a high-

intensity accelerator. The plan puts discovering Terascale physics with the LHC and the ILC as Fermilab's highest priority. While supporting ILC development, the plan creates opportunities for exciting science at the intensity frontier.

If the ILC remains near the Global Design Effort's technically driven timeline, Fermilab would continue neutrino science with the NOvA experiment, using the NuMI (Neutrinos at the Main Injector) proton plan, scheduled to begin operating in 2011. If ILC construction must wait somewhat longer, Fermilab's plan proposes SNuMI, an upgrade of NuMI to create a more powerful neutrino beam.

If the ILC start is postponed significantly, a central feature of the proposed Fermilab plan calls for building an intense proton facility, Project X, consisting of a linear accelerator with the currently planned characteristics of the ILC combined with Fermilab's existing Recycler Ring and the Main Injector accelerator. The major component of Project $\mathrm{X}$ is the linac. Cryomodules, radio-frequency distribution, cryogenics and instrumentation for the linac are the same as or similar to those used in the ILC at a scale of about one percent of a full ILC linac.

Project X's intense proton beams would open a path to discovery in neutrino science and in precision physics with charged leptons and quarks. World-leading experiments would allow physicists to address key questions of the Quantum Universe: How did the universe come to be? Are there undiscovered principles of nature: new symmetries, new physical laws? Do all the particles and forces become one? What happened to the antimatter?

Building Project X's ILC-like linac would offer substantial support for ILC development by accelerating the industrialization of ILC components in the U.S. and creating an engineering opportunity for ILC cost reductions. It offers an early and tangible application for ILC R\&D in superconducting technology, attracting participation from accelerator scientists worldwide and driving forward the technology for still higher-energy accelerators of the future, such as a muon collider.

To prepare for a future decision, the Fermilab Steering Group recommends that the laboratory seek R\&D support for Project X, in order to produce an overall design of Project X and to spur the R\&D and industrialization of ILC linac components needed for Project X. Advice from the High Energy Physics Advisory Panel will guide any future decision to upgrade the Fermilab accelerator complex, taking into account developments affecting the ILC schedule and the continuing evaluation of scientific priorities for U.S. particle physics.

Fermilab should also work toward increased resources for longer-term future accelerators such as a muon collider, aiming at higher energies than the ILC would provide.

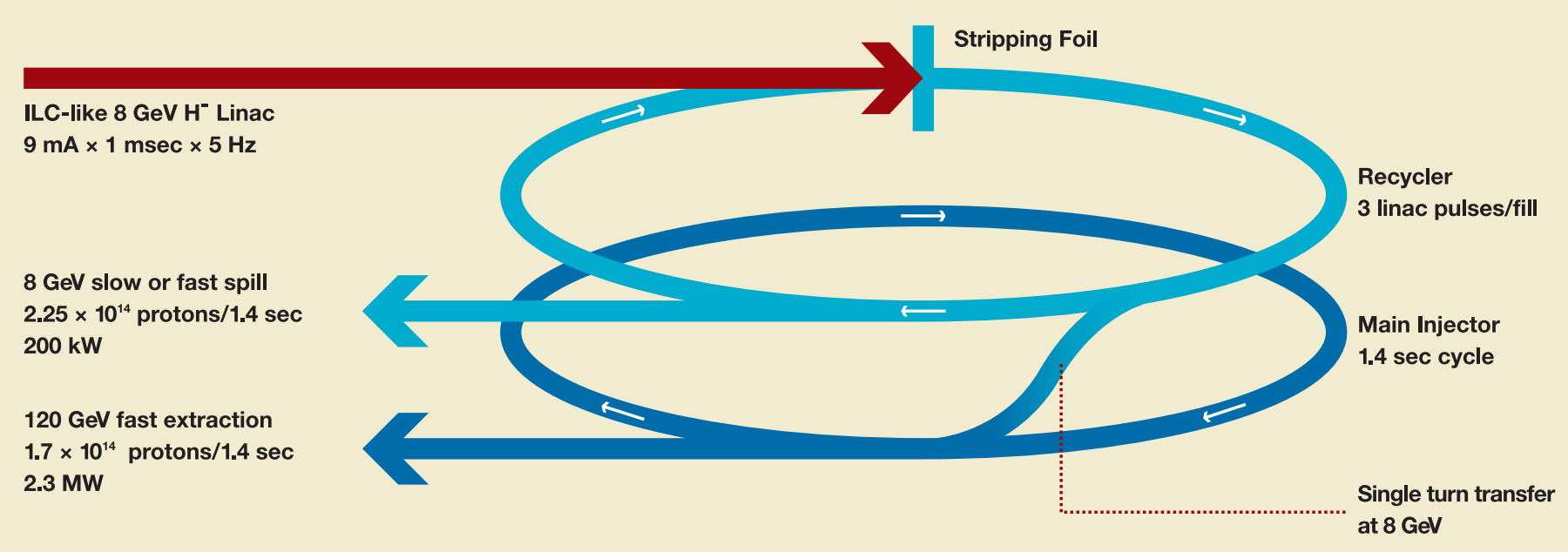




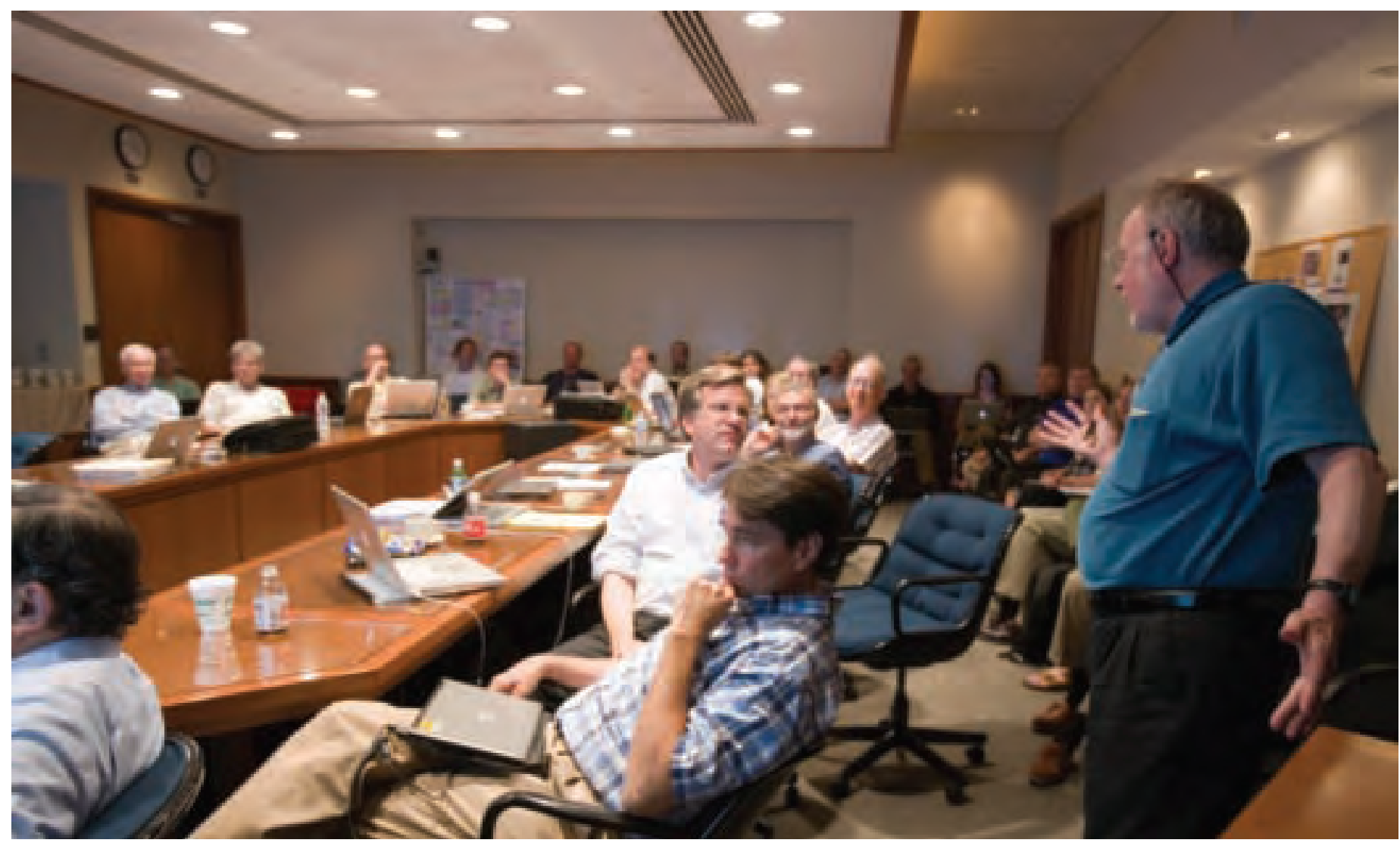

The Steering Group adopted a number of guidelines in forming the plan.

\section{Guidelines}

1. The LHC program is our most important near-term project given its broad science agenda and potential for discovery. It is essential to support the physics analysis, computing, and accelerator and detector upgrades.

2. The particle physics community's highest priority for investment toward the future is the ILC, based on our present understanding of its potential for breakthrough science. Fermilab will continue to participate vigorously in the international $\mathrm{R} \& \mathrm{D}$ program for the ILC and to be one of the leaders in the global ILC effort. The laboratory will strive to make the ILC at Fermilab a reality by accomplishing the preparatory work required for the U.S. to bid to host the ILC.

3. There is a need for an intermediate science program in case the timeline for the ILC is stretched out. This program will be an opportunity to do exciting physics that complements discoveries at energy frontier facilities and to make further progress on ILC technology. The program should provide great discovery potential, support ILC $\mathrm{R} \& \mathrm{D}$ and industrialization as well as R\&D on future accelerators beyond the ILC and the LHC. It should strengthen ties with the university community and with other laboratories. The plan must be robust and flexible.

4. Fermilab will continue a phased program of particle astrophysics including dark matter and dark energy. The program will allow complementary discoveries to those expected at the accelerator-based particle physics programs. These nonaccelerator-based efforts are outside the Steering Group's charge and are not included in the plan. 
The Steering Group recommends the following plan for the accelerator-based particle physics program at Fermilab.

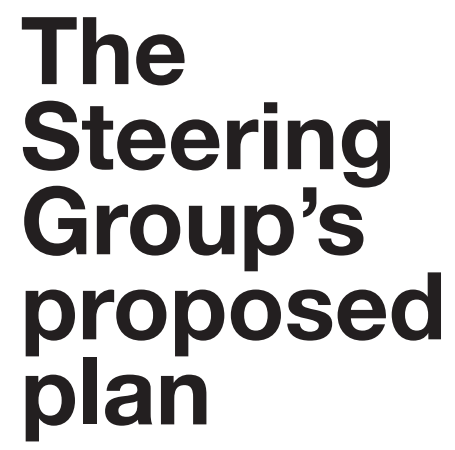

- Fermilab's highest priority is discovering the physics of the Terascale by participating in the LHC, being one of the leaders in the global ILC effort, and striving to make the ILC at Fermilab a reality.

- Fermilab will continue its neutrino program with NOvA as a flagship experiment through the middle of the next decade.

- If the ILC remains near the timeline proposed by the Global Design Effort, Fermilab will focus on the above programs.

- If the ILC departs from the GDE-proposed timeline, in addition Fermilab should pursue neutrino-science and precision-physics opportunities by upgrading the proton accelerator complex.

- If the ILC start must wait for a couple of years, the laboratory should undertake the SNuMI project. ${ }^{1}$

- If the ILC postponement would accommodate an interim major project, the laboratory should undertake Project $X$ for its science capability and ILC alignment. $^{2}$

- If the ILC is constructed offshore, in addition Fermilab should pursue neutrinoscience and precision-physics opportunities by upgrading current proton facilities while supporting the ILC as the highest priority.

- The laboratory should undertake SNuMI at a minimum.

- Alternatively, the laboratory should undertake Project $X$ if resources are available and ILC timing permits.

- In all scenarios,

- R\&D support for Project $X$ should be started now, with emphasis on

- expediting R\&D and industrialization of ILC cavities and cryomodules,

- overall design of Project X.

- R\&D for future accelerator options concentrating on a neutrino factory and a muon collider should be increased. ${ }^{3}$

- The laboratory should support detector R\&D and test-beam efforts for effective use of future facilities. 


\section{Chapter 2 Fermilab and the Quantum Universe}

0. What is the origin of mass for fundamental particles?

1. Are there undiscovered principles of nature: new symmetries, new physical laws?

2. How can we solve the mystery of dark energy?

3. Are there extra dimensions of space?

4. Do all the forces become one?

5. Why are there so many kinds of particles?

6. What is dark matter? How can we make it in the laboratory?

7. What are neutrinos telling us?

8. How did the universe come to be?

9. What happened to the antimatter? 


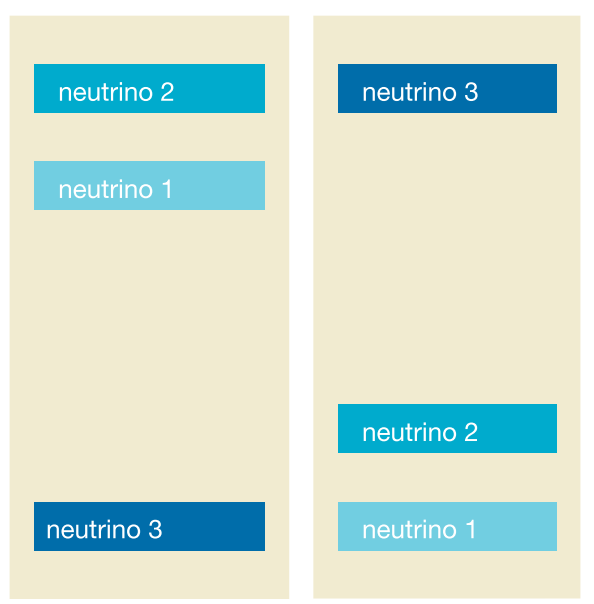

Neutrino mass hierarchy

How do neutrino masses stack up? The answer depends on how forces and matter become one-if they do. article physicists are on a 21st-century quest to answer profound questions about the universe. Powerful new scientific tools for particle physics and astrophysics now bring the answers to these compelling questions within reach. Along with astrophysical observations, particle accelerators offer different paths to the exploration of the physics of the Quantum Universe. At the energy frontier, the Large Hadron Collider at CERN and the proposed International Linear Collider will take physicists into a new "Terascale" energy region and the discoveries it holds. High-intensity accelerators offer another pathway to discovery by opening the door into the world of neutrinos and precision physics, where physicists expect they will also find answers to Quantum Universe questions.

The energy-frontier machines, the LHC and the proposed ILC, give physicists the means of discovering new symmetries and new physical laws, of finding extra dimensions of space and of finally penetrating the mystery of the origin of mass. Understanding the nature of dark matter will require energy-frontier accelerator programs to produce dark matter and analyze its properties. With the Tevatron running and the LHC nearing completion, the adventure of Terascale science has begun. Experiments at the LHC, built in Europe with U.S. participation, will provide a clear look at the Terascale. Hundreds of U.S. particle physicists will join collaborators from around the world in the largest scientific experiments ever conducted.

Physicists plan to build on the discoveries at the LHC with experiments at the proposed International Linear Collider. The ILC would allow experimenters to explore the new scientific landscape of the Terascale, revealing the properties of new phenomena and building the foundation for a clear and consistent understanding of this new energy terrain. Beyond this, precision measurements from the ILC could act as a telescope to reveal secrets from the much higher energies of the ultimate unification of forces and of matter.

Neutrino experiments, which have recently succeeded in detecting new physics, open their own window on unification, the question whether all the forces and particles of matter become one. Neutrinos have the unique potential to explain our cosmic beginnings from a process called leptogenesis. As part of Fermilab's world-class program in neutrino science, the laboratory has embarked on the NOvA experiment. NOvA will provide the first chance at determining the ordering of neutrino masses, a key piece of information for understanding the role of neutrinos in unification. The joint power of the Japanese T2K (Tokai to Kamioka) experiment and NOvA will be the first step toward experiments using high-intensity neutrino beams to detect the matter-antimatter properties of neutrinos that leptogenesis requires. Neutrino discoveries could link up with LHC or ILC discoveries of phenomena such as supersymmetry or with a charged-lepton-flavor-violation experiment, the morphing of one kind of charged lepton to another.

As the U.S. particle-physics community embarks on this global journey of discovery, the Particle Physics Project Prioritization Panel subpanel of the High Energy Physics Advisory Panel in 2006 laid out a roadmap for particle-physics research over the next decade in the United States. The P5 roadmap set priorities for U.S. particle physics aimed at maximizing the potential for discovery. Fermilab is strategically aligned with the P5 roadmap with a research program of

- energy-frontier physics starting with the Tevatron, continuing with the Large Hadron Collider, and following with the proposed International Linear Collider,

- accelerator-based neutrino physics,

- particle astrophysics including dark matter and dark energy.

The P5 roadmap charts a course for U.S. particle physics at a key moment in the life of the field. While accelerator-based particle physics is exciting and strong internationally, particle physics in the United States is confronting a very challenging period. By the end of the decade, the world-class programs at the Tevatron, the SLAC B Factory and Cornell's CESR will be complete. The contributions of U.S. facilities to global particle 
Did we all come from neutrinos?
Leptogenesis, from the Greek for "delicate origins," is the theory that all visible matter (stars, planets, people) comes from neutrinos. The cooling fireball of the big bang produced matter and antimatter directly, but in nearly equal amounts. Precise measurements of elementary particles show that the cosmic annihilation of matter and antimatter was almost complete, leaving not nearly enough leftover matter to form the billions of stars that we see today. Where did all this matter come from? Leptogenesis could be the answer.

The theory of leptogenesis starts with the observation that neutrinos are very different from other kinds of matter. Theorists postulate that neutrinos may be the only matter particles that are their own antiparticles. If so, it means that they obey a different set of rules with respect to the symmetry between matter and antimatter, or CP symmetry. Neutrinos also have superlight masses, which to physicists suggests a "see-saw" with superheavy partner neutrinos, not yet detected.

When theorists rerun the tape of the big bang introducing superheavy partner neutrinos that have nonstandard CP symmetry, the result is leptogenesis. The heavy neutrinos fall apart into light neutrinos, producing an excess of matter over antimatter. In the hot environment of the early universe, this excess is quickly passed along to all the particles that we are made of. If the theory of leptogenesis is correct, we owe our existence to neutrinos from the big bang.

physics will then come solely from the Main Injector at Fermilab for a neutrino physics program, and from a test-beam program for evaluating new and innovative detector concepts. In the U.S., an era of world-leading accelerator-based science at the energy frontier will come to an end. On the other hand, the conclusion of research at these U.S. accelerator facilities provides an opportunity to redirect resources toward hosting the ILC in the U.S. in order to continue the nation's historical role as a world leader in the science of particle physics.

Throughout Fermilab's history, the heart of the laboratory's scientific research has been the quest to solve the mysteries of the universe using energy-frontier particle accelerators. Because of its unique discovery potential and its significance for the national program, the ILC is Fermilab's highest priority for the future. Fermilab is committed to leadership in the international effort to build the ILC as early as possible, and the laboratory is a strong contributor to the ILC's Global Design Effort.

Following the technology choice for the ILC in 2004, the Global Design Effort and the international ILC community produced a Reference Design Report in February 2007 and are currently preparing an engineering design, required for a decision to build the ILC, that will be complete in 2010 .

The "technically driven" timeline for the ILC, based on technical readiness to proceed with the project, calls for a decision to go forward with the new collider in 2010 and for an ILC construction start early in the next decade. The P5 Panel assumed such a timeline in developing the roadmap for U.S. particle physics. However, because factors other than technical feasibility may postpone the start of the ILC, it becomes necessary to carefully plan the U.S. particle-physics program both to secure the ILC and to continue to contribute to particle physics discovery during a possibly extended period before the ILC can open up new scientific horizons.

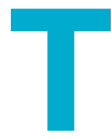
he goal of the Steering Group is to provide a Fermilab plan for scientific discovery in accelerator-based particle physics. In line with the P5 priorities, the plan represents a strategy to ensure the continuing U.S. capability to address the compelling questions of particle physics using the unique scientific potential of particle accelerators. The plan is flexible, offering options to address the scientific opportunities and challenges facing particle physics in the U.S. today. It keeps the ILC as the central feature of the Fermilab accelerator-based particle-physics plan and advances progress on technologies that will be needed for future frontier accelerators, such as a muon collider. The plan provides discovery opportunities should the timeline for ILC construction stretch out for any number of reasons: physics results, federal funding decisions, international 


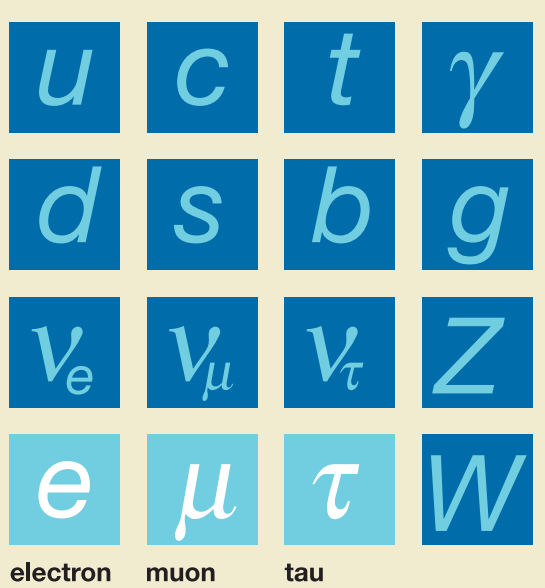

The charged leptons

Can one lepton change to another? If so, neutrino properties are pointing toward the unification of matter, the origin of all the elementary particles we know today from one single kind of particle at the big bang.
Unification and LFV

In the Standard Model, the weak interactions connect the three kinds of neutrinos to the three particles known as charged leptons: electron, muon and tau. Since experiments have discovered that neutrinos change from one kind to another, physicists wonder if the charged leptons do too. By producing huge numbers of muons in a controlled environment, experimenters hope to observe the direct conversion of a muon into an electron. This would be the first observation of lepton flavor violation outside the world of neutrinos.

These and other hints from data point toward matter unification, the idea that all of the charged leptons, neutrinos and quarks arose from a single kind of superparticle in the first instant of the big bang. Theorists find that when they put the ideas of unification and supersymmetry together, their models predict LFV for charged leptons at a rate that next-generation experiments could detect.

In models of unification, LFV is related to the process of leptogenesis. LFV with charged leptons is sensitive to different parts of the mechanism of leptogenesis from those accessible by neutrino experiments. An experimental program combining neutrino science with muon-to-electron conversion experiments and energy-frontier searches for supersymmetry would be a powerful probe of our unified origins.

agreements, site decisions for the ILC or other factors. The Steering Group's proposed plan sustains the potential for accelerator-based discovery in the U.S. both at the energy frontier with the ILC and with intense proton beams in the event of a delayed ILC. Crucially, the plan strengthens ties with university scientists and other laboratories and provides scientific training and education for hundreds of graduate students, the next generation of particle physicists.

Particle physics has become an interconnected global enterprise, with overarching scientific priorities defining regional and national plans. A plan for any specific laboratory must be formulated as part of a national program that is in turn coordinated with plans from other regions. Any new facility is likely to have competition elsewhere, and the scientific challenges dictate the need for strong research capabilities in all regions. The Fermilab Steering Group is a step in an integrated planning process - in the U.S. through P5 and HEPAP and then in a fully global context.

For U.S. particle physics, the decade ahead will bring great scientific opportunity and difficult challenges. Our questions for the universe could not be more profound or more compelling, especially because the means to address them are at last within reach. How the university and laboratory community comes together with government to meet the challenges and rise to the scientific opportunities is likely to shape the course of particlephysics research in the United States for a long time to come. In this context, Fermilab has a unique responsibility as the nation's primary particle physics user facility. The Fermilab Steering Group proposes a plan for the laboratory that is pragmatic, scientifically exciting and flexible enough to meet the challenges of a still-unfolding future and to provide for Fermilab's users the greatest possible opportunity for scientific discovery. 


\section{Chapter 3 Fermilab and the ILC}




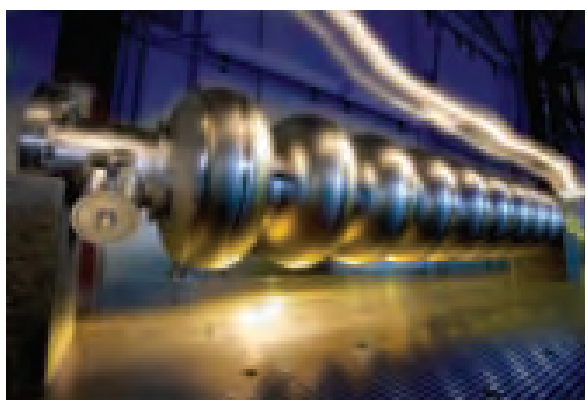

A superconducting ILC cavity

Credit: Fermilab Visual Media Services

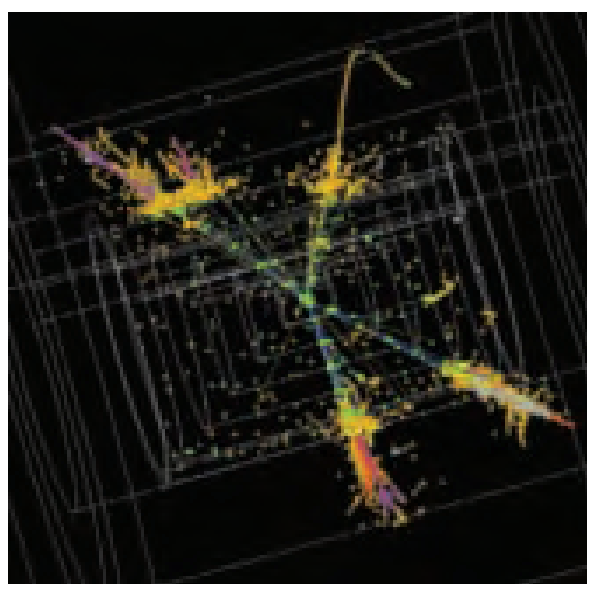

A simulation of the decay of a $Z+$ Higgs to four jets in an ILC detector Credit: Norman Graf
$\mathrm{T}$ he ILC promises extraordinary power in the study of the Terascale. The annihilation of an electron and its antiparticle, the positron, allows the understanding of collisions to an unparalleled level of detail and precision. As others have comprehensively documented', the ILC view of the Terascale, complementary to the LHC's perspective, makes the ILC an essential tool for unraveling new phenomena discovered at these extreme energies. It makes the ILC the top priority at Fermilab for a future global facility.

The ILC's opportunities for discovery have motivated the global particle physics community to come together in an effort to design the accelerator and its experimental program. The completion of the Reference Design Report in early 2007 and the structuring of a collaborative worldwide R\&D program represent successful community efforts. Fermilab has contributed strongly to this effort: the design of the accelerator; the development of superconducting radio-frequency, or SCRF, technology in the U.S.; the design of the physics and experimental program; the site studies necessary for hosting the ILC at or near Fermilab; and the establishment of a test-beam facility for the development of ILC detectors. The ILC and related SCRF efforts at Fermilab make up by far the laboratory's largest future program.

In the next phase of the ILC effort, Fermilab's aim is to be a leader in the global engineering design and in the development of the SCRF technology, steps necessary to reach a decision early in the next decade to build the ILC. Fermilab is building the required infrastructure and test facilities and is coordinating the national efforts in the development of SCRF technology, in collaboration with national and international partners in Europe, Asia and the U.S. To these efforts Fermilab brings strong engineering capability, accelerator physics expertise and technology development skills.

Innovative detectors will be key to exploiting the ILC physics opportunities. In general, an improvement in resolution of both tracking and calorimetric detectors over the present state-of-the-art detectors will allow experimenters to distinguish the signals of new physics from backgrounds much more efficiently. Fermilab has a strong instrumentation development effort in collaboration with laboratories and universities across the world. Just as important for the global ILC effort, Fermilab has developed and will operate a flexible high-energy test beam to provide a variety of particles and energies for testing detector technologies.

Fermilab's goal is to host the ILC. Geographically and geologically, the site is nearly optimal and could house the central facilities of the ILC, such as damping rings and experimental halls. Two important aspects of Fermilab's activities over the next three years are the study of the site and the design of conventional facilities necessary for the engineering design and working with the neighboring communities on issues associated with hosting the ILC in the region. Fermilab has vigorously collaborated with local residents over the last two years, first with the Community Task Force and currently with the ILC Citizens' Task Force and the Envoy Program. These activities will strengthen over the next three years of engineering design.

Finally, Fermilab is strengthening its engineering capabilities as the laboratory moves toward the design of global accelerators. Unlike the case of the detector community, which is accustomed to building detectors collaboratively across continents, much less collaboration has taken place in the development of global accelerators. The ILC is breaking new ground in this regard, and it is important that Fermilab have the strongest engineering cababilities and systems in place in order to lead in the integration of components produced around the world into a functioning accelerator.

The ILC is key to the future of U.S. particle physics and to Fermilab's future. 


\section{Chapter 4 Physics at the Intensity Frontier}




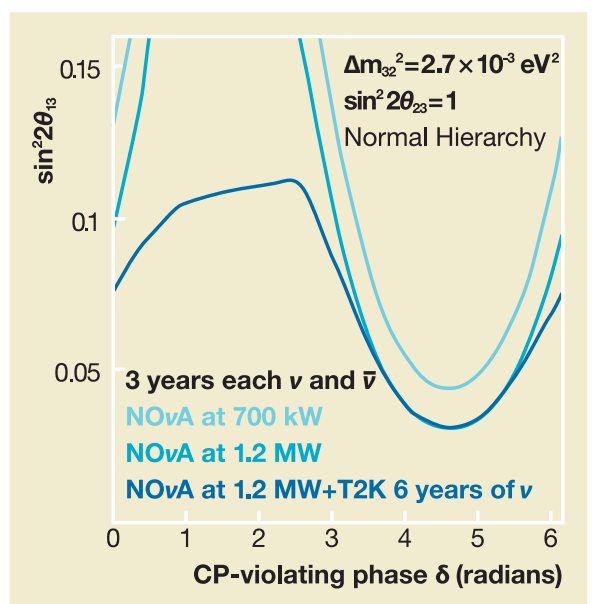

Sensitivity to mass hierarchy Ability of NOvA and NOvA plus T2K experiments to resolve mass hierarchy at 95 percent confidence level.
T he Standard Model of Particle Physics succeeds brilliantly at explaining the nature of the physical universe, but it leaves many open questions. Despite the development of myriad intriguing theories to address these questions, ultimately only experiment can light the way to discovery. In our own time, experiments at the energy frontier can search directly for new phenomena beyond the Standard Model. Alongside the compelling physics of Terascale explorations at the LHC and the ILC, another window on discovery has opened with the remarkable recent developments in neutrino science and with the ability to detect new physics phenomena in ultrarare events or in the small perturbations they induce in other processes. This chapter focuses on accelerator-based opportunities at the intensity frontier. Experiments in symmetry-violating processes and rare decays can provide windows into new mass scales of many thousands of TeV. Neutrino experiments may tell us about physics at even higher energies near unification or about an entirely new source of $\mathrm{CP}$ violation that may help explain the excess of matter over antimatter in the universe.

\section{Neutrino science}

An upgrade to the Fermilab proton complex could greatly enhance the laboratory's current world-class program of neutrino science by strengthening Fermilab's flagship program of long-baseline neutrino-oscillation experiments. It would provide for a next-generation experiment to discover $\mathrm{CP}$ violation in the leptonic sector, and consequently to explore leptogenesis as the source of matter-antimatter asymmetry in the evolution of the universe. The upgrade would also provide an opportunity for new, smaller-scale experiments using intense neutrino beams generated by $8 \mathrm{GeV}$ and $800 \mathrm{GeV}$ protons that would complement the long-baseline program.

\section{Long-baseline neutrino oscillations}

The Neutrino Scientific Assessment Group, convened by HEPAP and the Nuclear Science Advisory Committee, and a study group originally commissioned by Fermilab and Brookhaven National Laboratory have recently studied and documented the physics opportunities of long-baseline neutrino experiments. As laid out by NuSAG, this acceleratorbased program has as its primary goals to complete our understanding of neutrino mixing and oscillations, in particular to determine the ordering and splitting of the neutrino mass states, to measure the mixing angles and to determine whether there is $\mathrm{CP}$ violation in neutrino mixing. The study of $\mathrm{CP}$ violation in neutrino oscillations is especially compelling because $\mathrm{CP}$ violation in the leptonic sector may help explain the very fundamental problem of the matter-antimatter asymmetry of the universe through the process known as leptogenesis. Together, the Japanese T2K experiment and NOvA will begin to explore CP violation in neutrinos. Discovering the ordering of the neutrino mass states- the mass hierarchy - will help determine whether neutrino mass is related to the unification of the forces and whether neutrino oscillations violate $\mathrm{CP}$ symmetry. It may be key to interpreting the outcome of neutrinoless double-beta-decay experiments. Provided that neutrino mixing is large enough, the ability of the NOvA experiment to determine the ordering of the neutrino mass states makes the U.S. long-baseline neutrino program unique in the world.

Experiments to address these neutrino science goals will require both powerful beams and very large detectors, with the product of beam power and detector mass more than an order of magnitude larger than NOvA-generation experiments. Such "Phase II" experiments will require intense muon neutrino beams, regardless of detector technology and regardless of whether the detector has an off-axis or wide-band beam energy configuration. The

The long-baseline program could also unveil exotic effects due to sterile neutrinos, extra dimensions and dark energy. 


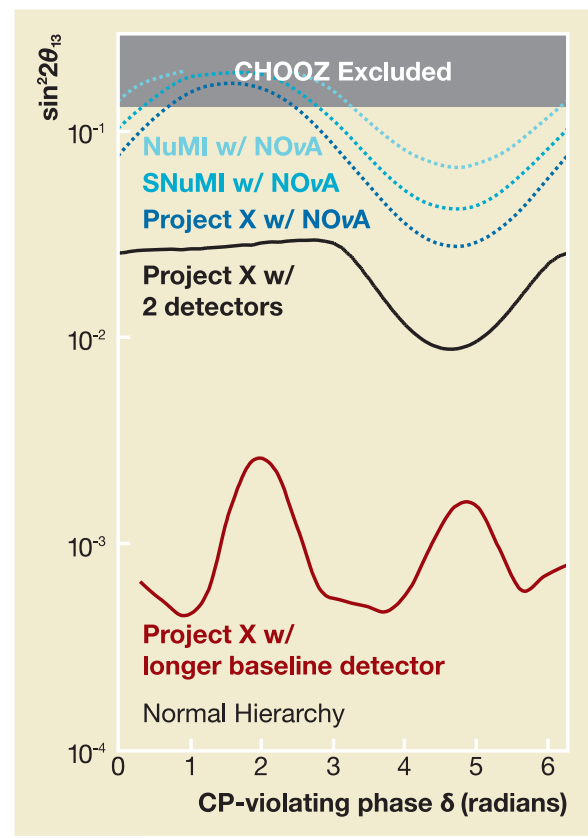

Sensitivity to mass hierarchy Ability to resolve mass hierarchy at 95 percent confidence level (dotted lines) and $3 \sigma$ (solid lines) of potential future Fermilab experiments. See details in Appendix C.

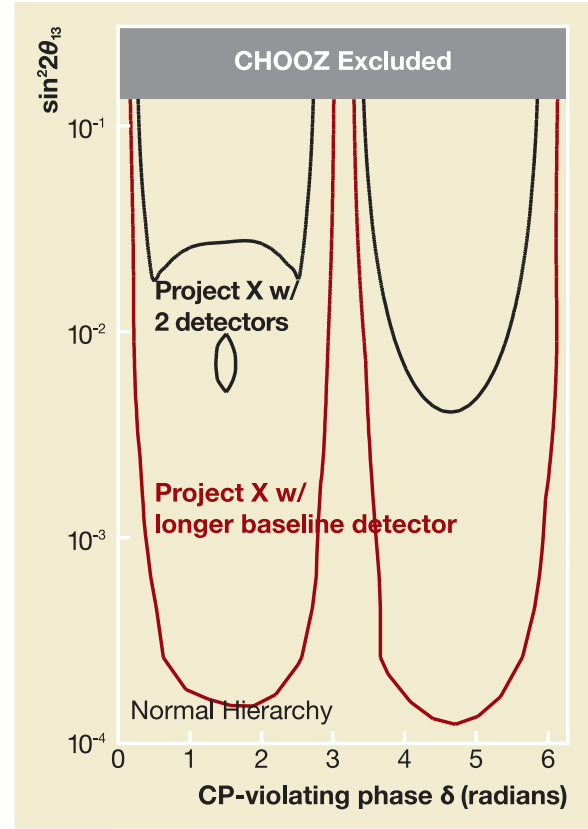

Sensitivity to CP violation

Sensitivity to $\mathrm{CP}$ violation at $3 \sigma$ confidence level of potential future Fermilab experiments. See details in Appendix C. discovery potential of these experiments will greatly benefit from higher proton beam power (thus higher neutrino flux) and greater flexibility of beam energy than is presently planned.

The proposed Project $\mathrm{X}$ would provide $2 \mathrm{MW}$ or more in the range of proton energy between $50 \mathrm{GeV}$ and $120 \mathrm{GeV}$. Compared to the NuMI proton plan for NOvA, it would supply approximately a factor of seven higher power at $50 \mathrm{GeV}$ and a factor of three higher power at $120 \mathrm{GeV}$. Project X's flexible beam energy and higher beam power, combined with the longer baselines of Phase II oscillation experiments such as those at the proposed Deep Underground Science and Engineering Laboratory, 1300 km from Fermilab, would confer impressive sensitivity to the neutrino mass hierarchy and CP violation.

A detector for a Phase II neutrino oscillation experiment, if located in the National Science Foundation's DUSEL, would also be a world-class detector for proton decay, addressing the question Do all the forces become one? This detector could also perform high-statistics studies of atmospheric neutrinos and carry out astrophysical searches including detection of relic-supernova neutrinos and neutrino bursts from supernovae in our galaxy and nearby.

The physics reach and competitiveness of the near-term NOvA experiment would also improve with SNuMI, an upgrade of NuMI that would increase $120 \mathrm{GeV}$ proton power to $1.2 \mathrm{MW}$. (SNuMI's beam power with $50 \mathrm{GeV}$ protons would be approximately $400 \mathrm{~kW}$.) SNuMI would support a neutrino program that would be both competitive and complementary to the T2K program based on the Japanese Proton Accelerator Research Complex. The SNuMI beam power is roughly 60 percent higher than that planned for Phase I of the J-PARC facility, and would remain competitive at least through the latter half of the next decade, depending on upgrades undertaken at J-PARC. Project $\mathrm{X}$ would markedly increase NOvA's sensitivity to the mass hierarchy and, with a Phase II experiment, would likely exceed the capabilities of the J-PARC facility (see Appendix C).

\section{Neutrino physics with $8 \mathrm{GeV}$ and $800 \mathrm{GeV}$ protons}

The Booster neutrino beam generated by $8 \mathrm{GeV}$ protons offers opportunities for neutrino studies beyond the existing experiments MiniBooNE and SciBooNE. In addition, experiments using high-energy neutrinos produced in a Tevatron fixed-target neutrino beam line would become possible if the Main Injector can provide sufficient $50-120 \mathrm{GeV}$ protons both to feed the long-baseline neutrino program and to generate $800 \mathrm{GeV}$ protons in the Tevatron. Some possible future experiments (see Appendix D) include:

Using $800 \mathrm{GeV}$ protons

- an experiment to precisely measure the weak mixing angle.

\section{Using $8 \mathrm{GeV}$ protons}

- an experiment to study low-energy neutrino interactions for neutrino-oscillation experiments such as MiniBooNE, NOvA and T2K, and to develop liquid-argon detector technology,

- an experiment to measure the strange quark contribution to the nucleon "spin."

The ability to conduct these experiments depends on the flexibility of the accelerator complex. Beam power at $8 \mathrm{GeV}$ is currently available for Booster neutrino experiments, because NuMI cannot use all Booster pulses for the long-baseline neutrino program. This situation will continue with the NOvA program. The SNuMI design, however, will be capable of using all Booster pulses for running NOvA at higher intensity, leaving none for neutrino experiments at the Booster. Alternatively, the Booster neutrino beam can run simultaneously with SNuMI at a tax of approximately 15 to 20 percent on the NuMI beam. Project $\mathrm{X}$, on the other hand, could deliver substantial $8 \mathrm{GeV}$ beam power (an order of magnitude more than is currently available) to experiments without a tax on NuMI. An 


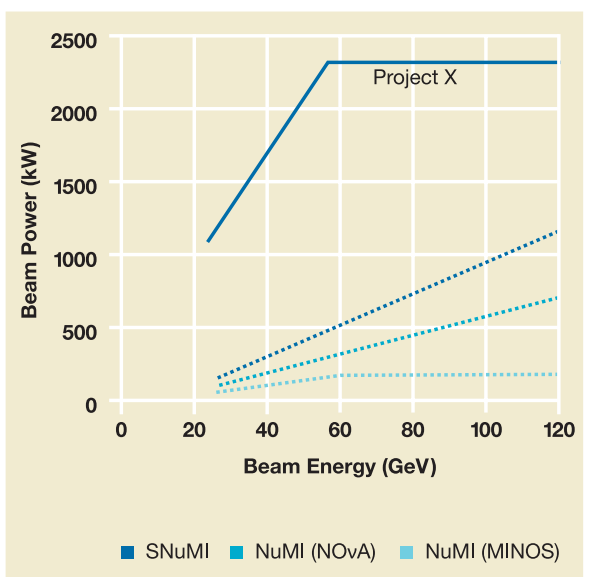

Proton beam power

Beam power versus beam energy for possible proton facilities at Fermilab.

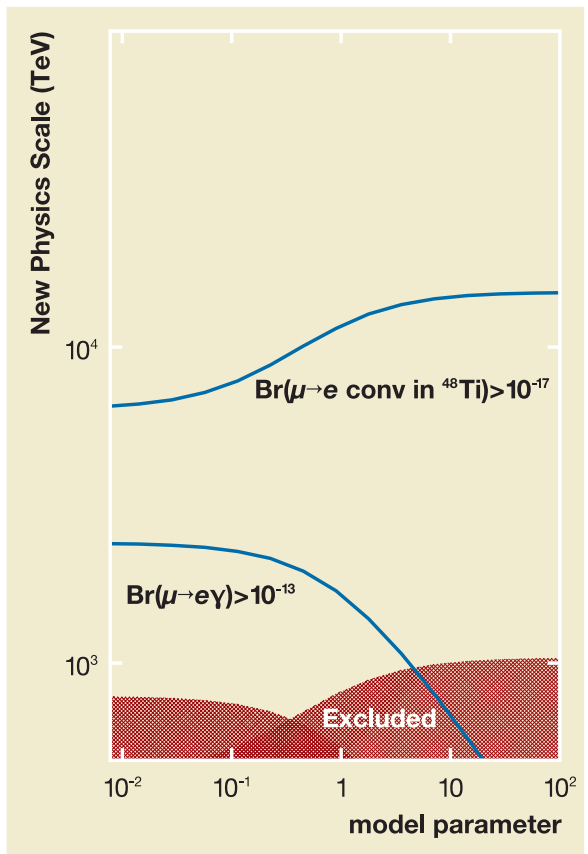

$\mu$-to-e conversion sensitivity Comparison of the sensitivity to lepton flavor violation of the MEG $(\mu \rightarrow e \gamma)$ experiment at a transition rate of $10^{-13}$ and a $\mu$-to-e conversion experiment with Fermilab Booster at the rate of $10^{-17}$. Project $X$ could reach the rate of $10^{-18}$. See details in Appendix E. experiment with an $800 \mathrm{GeV}$ proton beam would impose approximately a five percent tax on NuMI for both Project X and SNuMI. Proton-source upgrades, particularly Project X, make possible a stronger neutrino-science program.

\section{Precision physics}

Ultraprecise experiments using high-intensity sources of muons and quarks provide unique discovery potential. These experiments would complement those at the LHC as well as those in the worldwide program of neutrino science and precision physics. Results from these experiments would provide essential clues for interpreting discoveries and their implications for the great questions of particle physics.

\section{Muons}

Lepton flavor violation was discovered in neutrino experiments, where the three flavors of neutrinos are observed to morph, or oscillate, into one another. Physicists do not know why LFV occurs or if it is related to the flavor violation seen with quarks or to new phenomena at the Terascale. A key question is whether LFV also occurs with the charged leptons: electron, muon and tau. Theoretical models that incorporate ideas such as unification, supersymmetry or heavy-neutrino mixing predict charged LFV at rates that could be within reach of new experiments. Combined with results from neutrinos and the LHC, these experiments could point the way to leptogenesis or unification.

A new experiment could search for the direct coherent conversion of muons into electrons in the field of a nucleus. This muon-to-electron conversion experiment could detect LFV decays even if they occur at $10^{-17}$ the rate of standard muon processes. It would probe several distinct LFV processes. If a signal is detected, a $\mu \rightarrow e$ conversion experiment could zero in on the new physics by repeated measurements with different nuclear targets. This experiment would have sensitivity to very high energy scales, beyond the direct reach of colliders.

The Muon-to-Electron-Gamma experiment at the Paul Scherrer Institute will soon begin to look for the LFV process $\mu \rightarrow e \gamma$, with predicted sensitivity at the $10^{-13}$ level. A $\mu \rightarrow e$ conversion experiment at the $10^{-17}$ level would have greater sensitivity to the $\mu \rightarrow e \gamma$ transition than MEG, and orders of magnitude better sensitivity for more general LFV processes. Other approaches to LFV using taus are not expected to have comparable sensitivity, due to the available flux of taus, which is much less than that of muons, and to the greater cleanliness of the muon experiment.

A $\mu \rightarrow e$ conversion experiment at Fermilab could be 10,000 times more sensitive than previous experiments. An intense $8 \mathrm{GeV}$ proton beam and the Accumulator and Debuncher rings, available after the end of antiproton production for the Tevatron collider program, would make this LFV search possible. The SNuMI accelerator upgrades would increase the total proton flux at $8 \mathrm{GeV}$, allowing a modest increase in beam power for the muon program while also increasing the beam power available to the neutrino program. Project X could increase the beam power available to the muon program by a factor of 10 . Exploiting this increased intensity and a reoptimized muon beam (e.g. decreased energy spread and transverse beam size) has the potential to further improve sensitivity beyond that possible with the SNuMI upgrades. 


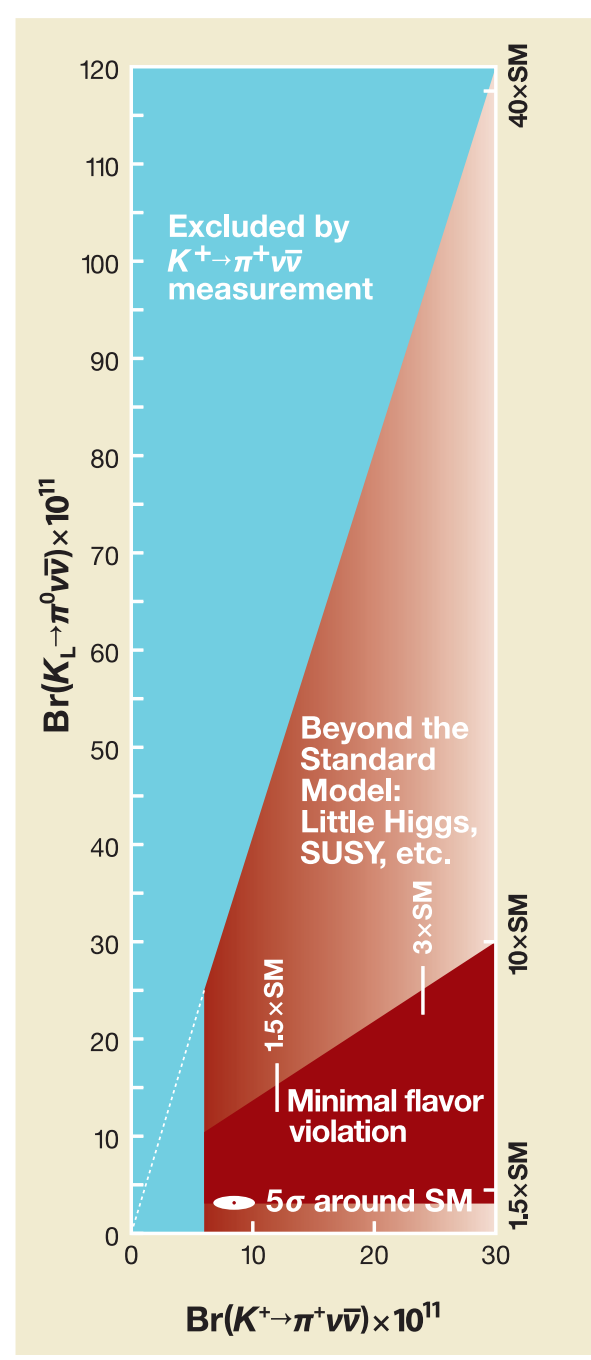

$K \rightarrow \pi v \bar{v}$ sensitivity

Project $\mathrm{X}$ experiments based on 1000 Standard Model events could probe Terascale physics with greater than five sigma sensitivity.

\section{Kaons}

Theories of Terascale physics typically predict new contributions to flavor-violating processes involving quarks. New particles predicted by Terascale physics are expected to have flavorviolating and $\mathrm{CP}$-violating couplings. Experiments at $B$ factories or elsewhere have unexpectedly found no clear signals of such contributions. These results favor theoretical models with minimal flavor violation. The data suggest a strategy of concentrating on rare processes that are as theoretically and experimentally clean as possible, to maximize the sensitivity to small contributions from new physics.

The ultrarare process $K \rightarrow \pi v \bar{v}$ is the most promising opportunity for implementing this new strategy. The neutral $K \rightarrow \pi v \bar{v}$ decay is extremely suppressed in the Standard Model and has not yet been observed. It is a clean, purely CP-violating process, with a Standard Model theoretical uncertainty no larger than two percent. A phased program at KEK and then J-PARC may eventually detect about 100 of these rare decays. The physics reviewed above shows the importance of a new experiment with the ultimate capability to detect about 1000 neutral decays, achieving a statistical error that approaches the theoretical uncertainty. Such an experiment would be even more powerful if combined with a precision measurement of charged $K \rightarrow \pi v \bar{v}$ decays, which are also highly suppressed in the Standard Model and have a modest theoretical uncertainty.

Such experiments would be sensitive to new sources of CP violation involving quarks. They would also be sensitive to flavor-violating effects from new particles, even in cases where the only source of CP violation is the CKM phase of the Standard Model. Either way, rare kaon decays offer a unique window on these phenomena. For example, if superpartner particles are discovered at the LHC, kaon experiments could address such fundamental questions as distinguishing among different mechanisms for the breaking of supersymmetry.

Other rare kaon-decay modes offer opportunities for major surprises. They include possible detection of the lepton-flavor-violating decays $K \rightarrow \pi \mu e$ or $K \rightarrow \mu e$, and exotic decays of kaons into axions or gravitons.

The high-intensity $8 \mathrm{GeV}$ proton facilities and the Tevatron Stretcher concept described in the next chapter represent a potential for a breakthrough in ultrarare kaon-decay experiments. They would provide kaon beams at Fermilab of unprecedented purity and intensity. Discovery sensitivities would benefit from increased kaon beam power. Project X's ability to optimize kaon beam characteristics would simplify the experiments and reduce technical risk.

\section{Charm and hyperon physics with antiprotons}

Fermilab operates the world's most intense antiproton source, a distinction it will continue to hold even after the planned 2014 startup of the Facility for Antiproton and Ion Research in Germany. The anticipated shutdown of the Tevatron collider program presents the opportunity for a world-leading low- and medium-energy antiproton program capable of studying a range of physics questions with unequaled sensitivity: hyperon $\mathrm{CP}$ violation and rare decays, charm mixing, the charmonium spectrum and recently discovered nearby states, and CPT and antimatter-gravity tests with antihydrogen. 


\section{Summary}

At the intensity frontier, the fields of neutrino science and precision physics offer promising pathways to physics breakthroughs not accessible to the LHC, the proposed ILC or nonaccelerator physics. In neutrino science:

- On the near horizon, NOvA will be the only experiment with sensitivity to the ordering of neutrino mass.

- The joint power of the Japanese T2K experiment and NOvA will begin to explore CP violation in neutrinos.

- In the post-NOvA era, a longer-baseline neutrino program with Project $\mathrm{X}$ and a very large detector would have unique capabilities to resolve neutrino-mass ordering. This program would remain unique even in the presence of the contemplated Japanese program with a very large detector in the Kamioka mine, fed by a multi-MW proton beam from J-PARC.

- By making possible $2 \mathrm{MW}$ proton beams at any energy between 50 and $120 \mathrm{GeV}$, Project $\mathrm{X}$ would create the flexibility to have excellent neutrino physics reach with either wide-band or off-axis beams.

- Project X, with a very large detector in the proposed DUSEL, would greatly enhance neutrino science.

- A post-T2K Japanese neutrino oscillation program would work at a different energy and a different baseline from its U.S. counterpart. Together, these two programs could make discoveries not accessible to either one alone.

In precision physics:

- Lepton flavor violation in muon decays offers a new window on physics beyond the Terascale, including leptogenesis and unification.

- A muon-to-electron conversion experiment at Fermilab would have unprecedented sensitivity and provide the ability to zero in on the origins of lepton flavor violation.

- New sources of flavor and CP violation predicted by Terascale physics have yet to be detected. Rare kaon decays offer a theoretically clean method of searching for these phenomena.

- Project X would provide the opportunity for an experiment to detect 1000 neutral and charged $K \rightarrow \pi v \bar{v}$ decays, offering a unique level of clean sensitivity to flavor- or CP-violating effects from Terascale physics.

What are neutrinos telling us? How did the universe come to be? Are there undiscovered principles of nature? What happened to the antimatter? Do all the forces become one?

An intensity-frontier program that provides unique experiments to address these profound questions of 21st-century physics would serve many scientific users. It would prepare future generations of U.S. particle physicists to exploit the potential of acceleratorbased scientific opportunities in the U.S. and worldwide. At the intensity frontier, Project X would help pave the way to the extremely powerful energy- and intensity-frontier facilities - a neutrino factory and a muon collider — of the long-term future beyond the ILC. 


\section{Chapter 5 Facilities for the Intensity Frontier}


The Steering Group considered a variety of accelerator facilities and programs using the following criteria:

- Support for physics research goals,

- Effective use of accelerator assets freed up at the end of Tevatron operations,

- Alignment with the ILC R\&D program,

- Potential for achievement over the next decade.

Twelve facilities received consideration using some or all of these criteria. Appendix G sorts those facilities not described in this chapter based on relevance to proton- or electron-based programs. This chapter describes the facilities that would support neutrino science and precision physics at the intensity frontier. The facilities include an intense proton source and its injection to the existing rings at Fermilab for a variety of programs.

There are two approaches to making an intense proton source at Fermilab. The first and more powerful approach would replace the present injection complex with Project X, an ILC-like linac capable of accelerating intense beams of either protons or electrons. Project X would use the linac, coupled to the Recycler and Main Injector, to produce much higher proton intensities in the range between $8 \mathrm{GeV}$ and $120 \mathrm{GeV}$ than the present complex. SuperNuMI, the second approach, would reconfigure the existing accelerator complex. Project X would be much more capable than SNuMI, which would leave the 35-year-old proton injection complex in place. SNuMI would provide less flexibility, involve more technical risk and have no alignment with the ILC— but it would also be less expensive and faster to implement than Project X. The Steering Group plan assumes a choice between SNuMI and Project X depending on the scope and longevity of the neutrino program and precision physics program, the desire to develop ILC technology on a real machine and the time available before construction of the ILC.

The table below represents the intensities of the present and future Fermilab accelerator complex using either SNuMI or Project X. The first three columns represent current performance and improvements now underway. The last two columns list SNuMI and Project X parameters. All columns are based on injecting beam from the existing $8 \mathrm{GeV}$ Booster, except for Project X, which eliminates the need for the Booster. While the table does not list any beam power availability at $8 \mathrm{GeV}$ in SNuMI, protons could be made available at this energy at the expense of availability at $120 \mathrm{GeV}$.

Possible evolution of proton availability at Fermilab

\begin{tabular}{|c|c|c|c|c|c|c|}
\hline & Now & Proton Plan & NOvA * & SNuMI & Project X & \\
\hline Batch Intensity (8 GeV) & $4.4 \times 10^{12}$ & $4.3 \times 10^{12}$ & $4.1 \times 10^{12}$ & $4.5 \times 10^{12}$ & $5.6 \times 10^{13}$ & protons/pulse \\
\hline Rep Rate & 7 & 9 & 12 & 13.5 & 5 & $\mathrm{~Hz}$ \\
\hline Protons/hour & $1.1 \times 10^{17}$ & $1.4 \times 10^{17}$ & $1.8 \times 10^{17}$ & $2.2 \times 10^{17}$ & $1.0 \times 10^{18}$ & \\
\hline Main Injector batches & 7 & 11 & 12 & 18 & 3 & \\
\hline MI batches to pbar target & 2 & 2 & 0 & 0 & 0 & \\
\hline MI Cycle Time & 2.4 & 2.2 & 1.33 & 1.33 & 1.4 & $\sec$ \\
\hline MI Beam Power (120 GeV) & 176 & 338 & 710 & 1169 & 2314 & $\mathrm{~kW}$ \\
\hline 8 GeV Beam Power (available) & 18 & 17 & 16 * & 0 & 206 & $\mathrm{~kW}$ \\
\hline Injection energy into 1st synchrotron & n 400 & 400 & 400 & 400 & 8000 & $\mathrm{MeV}$ \\
\hline
\end{tabular}

* NOvA column includes a potential upgrade of the Booster repetition rate to support simultaneous delivery of $\sim 2 \times 10^{20}$ protons per year at $8 \mathrm{GeV}$. NOvA itself requires Booster operations at $9 \mathrm{~Hz}$. 


\section{SuperNuMI (SNuMI)}

SNuMI uses antiproton facilities freed up at completion of the Tevatron collider program to develop a more intense proton source for NuMI. The Antiproton Accumulator would "momentum-stack" protons delivered from the Booster. The momentum-stacking process is inherently more efficient in its use of longitudinal phase space than the "slip-stacking" process that will be used during the NOvA era. This increased efficiency supports the higher proton throughput of SNuMI.

The SNuMI scheme momentum-stacks three Booster batches in the Accumulator and then transfers them to the Recycler. This process repeats six times via "boxcar" stacking in the Recycler. The accumulated 18 batches are transferred from the Recycler to the Main Injector in a single shot. Because the Booster cycles at $15 \mathrm{~Hz}$, the Recycler accumulation process takes 1.33 seconds. The accumulation process takes place while the Main Injector is accelerating, fast-extracting beam to the neutrino target and ramping down for a new load. A 1.33 second cycle time leads to a beam power of $1.2 \mathrm{MW}$. Since this scheme uses all available Booster cycles, no additional protons are available for an $8 \mathrm{GeV}$ program without diversion from the Main Injector. However, $\mathrm{SNuMI}$ is compatible with reconfigurations of the Debuncher ring and/or the Tevatron to support slow-spill programs at 8 or $120 \mathrm{GeV}$ respectively, and with the Tevatron high-energy neutrino facility.

SNuMI could probably be constructed over a two-to-three-year period following completion of Tevatron collider operations. While it would support some of the neutrinoscience and precision-physics goals, the SNuMI plan would require continued use of the existing $8 \mathrm{GeV}$ Booster and $400 \mathrm{MeV}$ Linac accelerators, which date from the 1972 start of beam operations at Fermilab. These accelerators' aging components have led to reliability issues over recent years. Thus, $\mathrm{SNuMI}$ entails some risk of operational down time or failures and does not invest in a longer-term program of experiments.

\section{Project X}

Project $\mathrm{X}$ is based on an $8 \mathrm{GeV}$ superconducting $\mathrm{H}^{-}$linac. The downstream $7 \mathrm{GeV}$ would use ILC cryomodules and radio frequency distribution systems, with perhaps some modifications in the transverse focusing arrangement and RF phase adjustment. Project X's front end draws heavily on technology developed by Argonne National Laboratory for a facility for rare isotope beams, the Advanced Exotic Beam Laboratory. Using the Recycler as a stripper and accumulator ring is the key element that allows the linac to run with the same beam parameters as the ILC. The linac operates at $5 \mathrm{~Hz}$ with a total of $5.6 \times 10^{13}$ $\mathrm{H}^{-}$ions delivered per pulse. They are injected into the Recycler using a standard $\mathrm{H}^{-}$stripping procedure. The total pulse length $(1 \mathrm{~ms})$ implies 100-turn injection. The injection

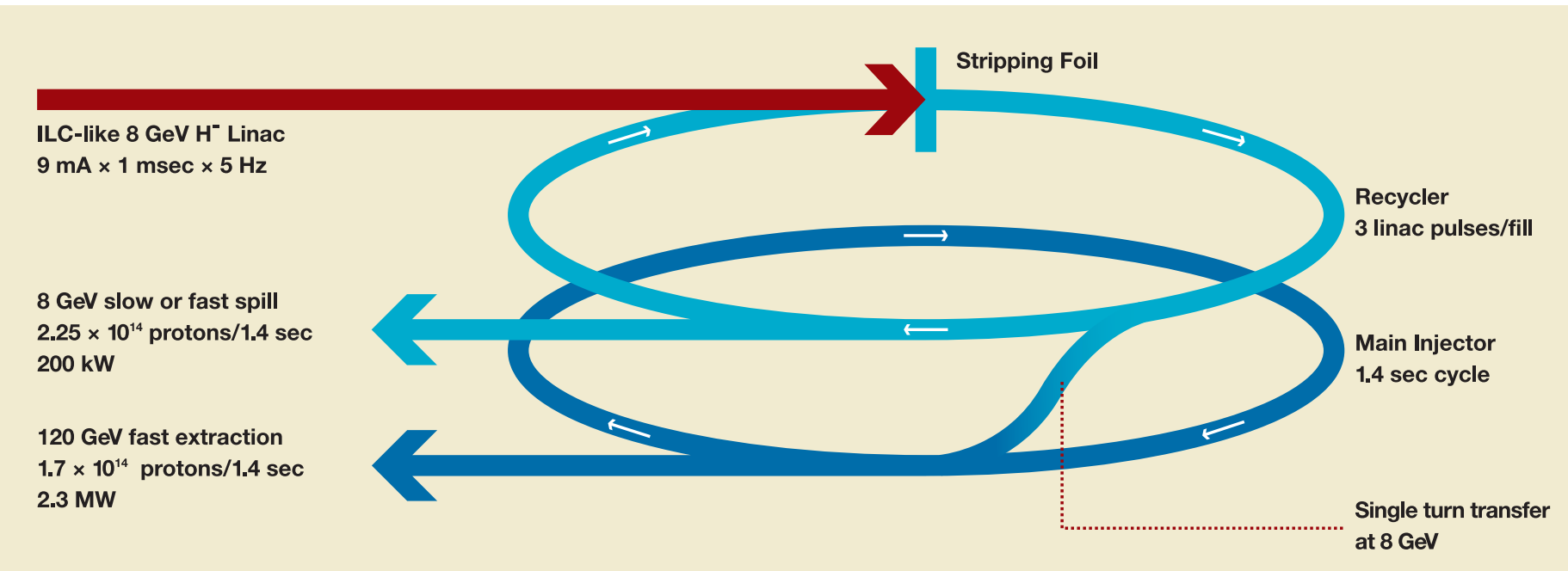


Following the $1 \mathrm{~ms}$ injection, the orbit moves off the stripping foil and circulates for $200 \mathrm{msec}$, awaiting the next injection. Following three such injections a total of $1.7 \times 10^{14}$ protons are transferred in a single turn to the Main Injector. These protons are then accelerated to $120 \mathrm{GeV}$ and fast extracted to a neutrino target. The Main Injector cycle takes 1.4 seconds, producing approximately $2.3 \mathrm{MW}$ of beam power at $120 \mathrm{GeV}$. At lower proton energies, Main Injector cycle times can be shorter, allowing a beam power above $2 \mathrm{MW}$ in the range of proton energy between $50 \mathrm{GeV}$ and $120 \mathrm{GeV}$. In parallel, because the loading of the Recycler only requires 0.6 seconds, up to four linac cycles are available for accumulation and distribution of $8 \mathrm{GeV}$ protons from the Recycler. Total available $8 \mathrm{GeV}$ beam power lies in the range of 100-200 kW, depending on the proton energy in the Main Injector. This is an order of magnitude higher than the proton power currently available. Different configurations of the Recycler could distribute this beam in any combination of fast or slow extractions required by the physics program. Project $\mathrm{X}$ is also compatible with reconfigurations of the Debuncher ring and the Tevatron to support slow spill programs at 8 or $120 \mathrm{GeV}$ respectively, and with the Tevatron High Energy Neutrino Facility. The Tevatron-based programs would come with a modest cost in protons delivered to the neutrino program at $120 \mathrm{GeV}$, because of the use of a Main Injector cycle to transfer beam to the Tevatron.

Taking full advantage of the increased beam power available from Project $\mathrm{X}$ would require changes to the Recycler, the Main Injector and the neutrino target. The Recycler would need a new $\mathrm{H}^{-}$injection system and probably measures to mitigate electron cloud effects, as well as a new (fast or slow) extraction system, and new RF systems. The Main Injector would need a new RF system and measures to mitigate electron cloud effects. Project $\mathrm{X}$ would require design and construction of a new neutrino target station to support 2.3 MW operations. Building a spur off the current NuMI beamline would permit directing a beam toward the DUSEL site.

The engineering design for Project $\mathrm{X}$ will produce a detailed cost estimate and construction plan. However, we expect that the accelerator portion of Project X would be comparable in size and scope to the Main Injector. Construction would take four to five years with a few hundred FTEs per year. It would be most effectively achieved as an interlaboratory collaboration centered at Fermilab.

\section{Existing rings}

Current operations at Fermilab use six rings: Booster, Accumulator, Debuncher, Recycler, Main Injector and Tevatron. The descriptions of Project X and SNuMI explain how the Main Injector would be used to produce an intense source of neutrinos, with nearly 10 times the intensity of the current source. Besides the Main Injector, the following examples show how an expanded program could use additional existing rings at Fermilab.

\section{Debuncher slow extraction}

The Antiproton Debuncher ring could provide an $8 \mathrm{GeV}$ slow-extraction facility with parameters appropriate for a muon-to-electron conversion experiment. Protons could be provided either from the Accumulator (SNuMI) or from the Recycler (Project X). An RF system within the Debuncher would confine the protons to a single bunch, and a slow extraction system would spill the beam over the 1.33-1.4 second cycle time. Operating with a single bunch, the circumference of the Debuncher creates a spill structure containing $\mathrm{a} \sim 100 \mathrm{~ns}$ pulse every $1.6 \mu \mathrm{s}$. With the SNuMI configuration, total delivered beam would be $1.35 \times 10^{13}$ every 1.33 seconds, with a corresponding 16 percent reduction in available protons at $120 \mathrm{GeV}$. If Project $\mathrm{X}$ were used to deliver protons, any number up to the full complement of $2.2 \times 10^{14}$ every 1.4 seconds could be made available with no impact on the $120 \mathrm{GeV}$ program. 


\section{Tevatron Stretcher}

Taking protons at $120 \mathrm{GeV}$ directly from the Main Injector, the Tevatron could be converted to a $120 \mathrm{GeV}$ "stretcher" ring to provide very high (>90 percent) duty factor beams for a variety of precision frontier experiments. The Tevatron Stretcher provides an independent program that could be used with or without the SNuMI or Project X intensity upgrades. However, the program would result in a tax on the Main Injector-based neutrino program.

A possible scenario would use two Main Injector cycles, at $3.75 \times 10^{13}$ protons per pulse, providing $7.5 \times 10^{13}$ protons in the Tevatron at $120 \mathrm{GeV}$. This beam is not accelerated, but rather is slow extracted over roughly 60 seconds. The duty factor would approach 95 percent. This scenario would deliver a total of about $3 \times 10^{19}$ protons in a year, representing approximately a five percent diversion of protons from the SNuMI or Project X neutrino program. Because the delivered intensity would be about a factor of 2.5 beyond the highest intensity ever stored in the Tevatron, the laboratory would need to address a number of intensity-related issues. In addition, this scheme would require the design and implementation of a $120 \mathrm{GeV}$ resonant extraction system.

The Tevatron Stretcher and associated extracted beam lines would require a one-to-two year construction period at an appropriate time following completion of Tevatron collider operations.

\section{Tevatron high-energy neutrino facility}

The Tevatron could operate at high intensity and high energy in fixed-target mode. The science program described in Chapter 4 would require a minimum beam energy of roughly $800 \mathrm{GeV}$, with a delivered intensity of at least $4 \times 10^{19}$ protons per year. The minimum cycle time of the Tevatron in fixed-target mode is about 40 seconds, establishing the basic per-pulse intensity requirement.

A possible scenario would resemble the Stretcher scheme described above. Two Main Injector cycles, at $3.75 \times 10^{13}$ protons per pulse, are transferred to the Tevatron at $120 \mathrm{GeV}$. This beam is accelerated and delivered to a neutrino target via a fast-spill mechanism. Based on a minimum Tevatron cycle time of 40 seconds, the scheme would deliver a total of about $4 \times 10^{19}$ protons per year, approximately a five percent diversion of protons from the SNuMI or Project X neutrino program. The same intensity issues associated with the Tevatron Stretcher would apply. Several other technical issues would also require resolution, including development of the fast-extraction scheme, mechanisms for loss control and collimation, recommissioning of the CZero high-intensity abort, and a reliability analysis.

Tevatron fixed-target operations would require one to two years to implement at an appropriate time following completion of Tevatron collider operations.

\section{Project $\mathrm{X}$ and the ILC}

Among the proton facilities that the Steering Group considered, Project X is unique in supporting ILC development at Fermilab. It would drive the initial stage of industrialization of cryomodules and provide experience with operating the linac as a system, advancing the ILC if a delay in a decision to construct slowed progress in industrialization.

\section{Industrialization}

The superconducting radio-frequency cryomodules are the most complex and expensive technical element of the ILC. Development of the national and institutional capability to build and test cryomodules with ILC specifications is a high priority of the GDE Americas Regional Team and of Fermilab in its bid to host the ILC. The DESY experience has shown that mastery of this technology requires significant infrastructure investments and a long learning curve for personnel.

Project X requires approximately 33 ILC-style cryomodules. In the current design, nine are "ILC-like" and 24 are "ILC-identical." These cryomodules provide acceleration between 
1.0 and $8.0 \mathrm{GeV}$. Production over a three-year period represents a significant advance over currently anticipated capabilities. However, such a production rate is below ILC requirements, so Project $\mathrm{X}$ would represent the initial phase of industrialization for ILC in the U.S. Full integration within an ILC industrialization plan requires more study.

\section{Operational experience and systems testing}

Project X could be configured to use the same beam parameters as the ILC $(9 \mathrm{~mA} \times 1 \mathrm{~ms}$ $\times 5 \mathrm{~Hz}$ ). The linac design calls for $31.5 \mathrm{MV} / \mathrm{m}$ but could operate successfully at lower gradients. The RF generation and distribution system would be the same as the ILC's, giving valuable experience with the klystrons, modulators, couplers, and cryomodules under operational conditions. The focusing arrangement, i.e. distribution of quadrupoles through the cryomodules, is somewhat different from the ILC's between $1 \mathrm{GeV}$ and $5 \mathrm{GeV}$.

Operation of the linac with electrons is also under study. It would require a mechanism to provide appropriate phasing of the cavities to compensate for the fully relativistic state of the electrons. Ferrite-based vector modulators, currently under development, could provide this capability. Operation with electrons at the full ILC specification would provide important understanding of higher-order modes and associated loads on the cryogenic system.

\section{Test beam for detector development}

Project X would substantially increase the capabilities of the $120 \mathrm{GeV}$ test-beam program and would support the laboratory's test-beam infrastructure through the construction of new beamlines driven by the $8 \mathrm{GeV}$ linac. These new test beams could provide $8 \mathrm{GeV}$ protons and electrons with the exact ILC beam-time structure, of interest to the ILC detector community for evaluation of readout strategies and low-energy calorimeter performance.

\section{Project $\mathrm{X}$ and longer term facilities}

The high-power $8 \mathrm{GeV}$ beam of Project $\mathrm{X}$ would support a program aimed at the development and demonstration of technologies required for muon-based storage rings such as a neutrino factory or muon collider.

\section{Summary}

The Steering Group identified two options that would provide significant enhancements to proton intensity in support of neutrino-science and precision-physics programs at Fermilab. Both options allow use of the Debuncher ring and/or the Tevatron to deliver beams in the range between $8 \mathrm{GeV}$ and $800 \mathrm{GeV}$. The SNuMI project is based on reusing existing antiproton rings for proton accumulation. SNuMI would support the delivery of about 1.2 MW of $120 \mathrm{GeV}$ proton beam power onto a neutrino target and could provide 8, 120 or $800 \mathrm{GeV}$ beams for precision physics programs via modest diversion of protons from the neutrino program. Project $\mathrm{X}$ is based on a new superconducting $8 \mathrm{GeV}$ linac. Project X would support more than $2 \mathrm{MW}$ of protons between $50 \mathrm{GeV}$ and $120 \mathrm{GeV}$ on the neutrino target, simultaneous with $200 \mathrm{~kW}$ of beam power at $8 \mathrm{GeV}$. The $8 \mathrm{GeV}$ beams could support a variety of precision physics experiments at 8,120 or $800 \mathrm{GeV}$. Fermilab could implement SNuMI within a couple of years and Project X in four to five years. Project X features significant technology alignment with ILC which would continue to develop U.S. industrial capabilities. 


\section{Chapter 6 Beyond the ILC and the LHC}




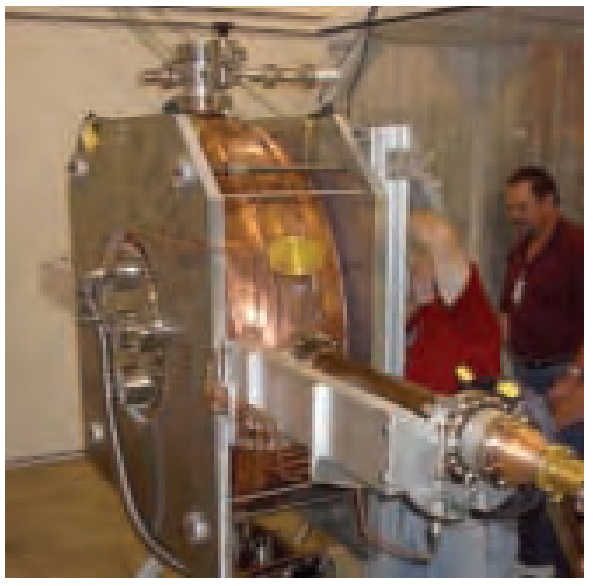

Muon Collider R\&D RF cavity being prepared for tests at Fermilab. Credit: Yagmur Torun
$\mathbf{T}$ he Steering Group examined the steps necessary to explore higher-energy colliders at Fermilab that might follow the ILC or that might be needed should the results from LHC point toward a higher energy than that planned for the ILC. Steps to explore higher-energy hadron and $e^{+} e^{-}$colliders are currently underway at other laboratories, with results expected within five years. The exploration of a muon collider is a far different matter and will require considerable attention and significantly increased resources.

\section{LHC energy upgrade}

Magnet technology needed to upgrade the LHC to $21 \mathrm{TeV}$ center-of-mass energy is currently under development as part of the LHC Accelerator Research Program, or LARP, and of the DOE base funding for magnet technology development. This technology should be ready for application in about five years.

\section{Very Large Hadron Collider}

Likewise, the basic technology that could support construction of a VLHC will be in hand on a five-year time scale should it be needed. Detailed magnet development would need to follow a reanalysis of the energy and optimum size of the collider once the physics objectives clarify. Luminosity will be a challenge if it is to increase beyond that planned for the LHC as required to follow the energy dependence of the physics cross section.

\section{Compact Linear Collider}

The current CERN midterm plan includes efforts to demonstrate by 2010 the CLIC two-beam accelerator concept using X-band technology for an $e^{+} e^{-}$collider up to $3 \mathrm{TeV}$. The U.S. High-Gradient Collaboration is studying technologies that may provide an alternative approach to a multi-TeV $e^{+} e^{-}$collider.

\section{Muon collider}

In contrast to the situation for electron and hadron colliders, demonstrating the viability of a muon collider will require many steps:

- exploration of various end-to-end conceptual designs,

- a specialized proton driver,

- various targeting, capture and phase-rotation schemes,

- various possible six-dimensional (6D) ionization cooling configurations,

- various methods of acceleration to high energy of the cooled muons,

- storage ring designs,

- detector configurations.

Each of these steps (see Appendix $\mathrm{H}$ for details) may involve development of more than one technology. Given the many unknowns, especially in mastering 6D ionization cooling, it is not possible to state even a technically limited schedule with any precision. However, a significant evaluation of cooling and other feasibility items might be carried out in approximately five to seven years. A rough comparison with the U.S. ILC development intensity prior to the International Technology Recommendation Panel decision would indicate the need for a minimum of $\$ 20 \mathrm{M}$ annually and $100 \mathrm{FTE}$ of appropriate skills in the U.S.

\section{Summary}

The Steering Group recommends a strengthening of the R\&D program for future accelerators such as a muon collider over the next five years independent of the ILC timeline. A construction start for the ILC early in the next decade would dictate reevaluation and adjustment of the effort as appropriate. If the ILC were built offshore, and if a satisfactory cooling method and a concept design for the collider system have emerged, the muon collider effort could rapidly ramp up. 


\section{Chapter 7 A Fermilab Plan for Discovery}




\section{The Steering Group's proposed plan}

The Steering Group recommends the following plan for the accelerator-based particle physics program at Fermilab.

- Fermilab's highest priority is discovering the physics of the Terascale by participating in the LHC, being one of the leaders in the global ILC effort, and striving to make the ILC at Fermilab a reality.

- Fermilab will continue its neutrino program with NOvA as a flagship experiment through the middle of the next decade.

The Steering Group plan gives the highest priority to energy-frontier physics with the LHC and the ILC, where experiments will search directly for the physics of the Terascale, addressing the most compelling questions of 21st-century particle physics. Fermilab is the U.S. host laboratory for the CMS experiment at the LHC and a lead laboratory for LHC accelerator development in the U.S.

The ILC's opportunities for discovery have brought the global physics community together to design the proposed new accelerator. Fermilab aims to be one of the leaders in worldwide ILC design and engineering, with the goal of hosting the ILC at Fermilab. Fermilab will pursue the most vigorous possible program of ILC research and development, communication and community outreach toward that goal. Fermilab expects to play a key role even if the ILC is built offshore.

Neutrino science opens another window on the key questions of particle physics. Fermilab will continue to build on the laboratory's world-class neutrino program by constructing the NOvA experiment, a more powerful successor to MINOS, to begin operating in 2011.

- If the ILC remains near the timeline proposed by the Global Design Effort, Fermilab will focus on the above programs.

- If the ILC departs from the GDE-proposed timeline, in addition Fermilab should pursue neutrino-science and precision-physics opportunities by upgrading the proton accelerator complex.

- If the ILC start must wait for a couple of years, the laboratory should undertake the SNuMI project.

- If the ILC postponement would accommodate an interim major project, the laboratory should undertake Project $X$ for its science capability and ILC alignment.

- If the ILC is constructed offshore, in addition Fermilab should pursue neutrinoscience and precision-physics opportunities by upgrading current proton facilities while supporting the ILC as the highest priority.

- The laboratory should undertake SNuMI at a minimum.

- Alternatively, the laboratory should undertake Project $X$ if resources are available and ILC timing permits.

If the ILC is delayed, the Steering Group's plan keeps Fermilab and U.S. particle physics on the pathway to discovery in the domain of neutrinos and precision physics, while advancing the technology of the ILC for the energy frontier. If ILC construction must wait, the plan proposes $\mathrm{SNuMI}$, an upgrade of the current $\mathrm{NuMI}$, to pursue neutrino science with a more powerful neutrino beam. If the ILC start is postponed significantly, the Steering Group proposes Project X, an intense proton-beam facility: a linear accelerator with the planned characteristics of the ILC at about one percent of the ILC's length, combined with existing Fermilab accelerator rings. 
Project $\mathrm{X}$ at Fermilab would make possible a broad range of intensity-frontier experiments to pursue the fundamental questions of particle physics via the pathways of neutrino science and precision physics. The potential depth and diversity of such an experimental program make the Steering Group's plan both flexible and scientifically compelling. While the plan proposes the outline of a world-class neutrino science and precision physics program at Fermilab, each experiment would be judged on its physics merits at the appropriate time through the laboratory and HEPAP advisory process. Besides providing unique opportunities for discovery, Project $\mathrm{X}$ would align with the development of the ILC. Its ILC-based technology would spur U.S. industrialization and reduce costs while creating a system test for key ILC components.

\section{- In all scenarios,}

- R\&D support for Project $X$ should be started now, with emphasis on - expediting R\&D and industrialization of ILC cavities and cryomodules, - overall design of Project X.

- R\&D for future accelerator options concentrating on a neutrino factory and a muon collider should be increased.

- The laboratory should support detector R\&D and test-beam efforts for effective use of future facilities.

To prepare for a future decision, the Steering Group recommends that Fermilab immediately seek R\&D support for Project $\mathrm{X}$ in order to develop an overall design. Project $\mathrm{X}$ could create a pathway toward a neutrino factory to produce the intense neutrino beams that would be needed for neutrino-oscillation and CP-violation experiments in case $\sin ^{2} 2 \theta_{13}$ is extremely small. A neutrino factory could also be a crucial step along the way to regaining the energy frontier in the U.S. by way of a muon collider, relying upon Project $\mathrm{X}$ as a muon source, followed by muon cooling.

\section{Summary}

The LHC and the ILC have the highest priority in the Steering Group's plan. In the event of a delay in the timeline for the ILC, an intense proton-beam facility such as Project X would offer a world-class experimental program in the U.S. and support the effort to host the ILC in the U.S. A physics program with an intense proton source offers strong opportunities for discovery, following alternate pathways to those offered by the LHC and the ILC for answering Quantum Universe questions. Its experiments would serve many scientific users and educate future generations of U.S. particle physicists. Project $X$ would align with the early phases of muon-collider development, starting the field on the path to the energy frontier beyond the ILC. The potential breadth, depth, scale and diversity of the science program, its supporting role for the ILC and its implications for future energy-frontier accelerators make the Steering Group plan flexible, robust and scientifically compelling. 


\section{Appendix}

33 Appendix A

Steering Group charge

34 Appendix B

Fermilab and the ILC

36 Appendix C

The international neutrino program

38 Appendix D

Neutrino science with $8 \mathrm{GeV}$ and $800 \mathrm{GeV}$ protons

40 Appendix E

Muon-to-electron conversion in nuclei

41 Appendix F

Precision physics experiments with kaon beams

42 Appendix G

Facilities considered

43 Appendix $\mathrm{H}$

Steps toward a muon collider 


\section{Steering Group charge}

In his remarks to HEPAP, Under Secretary

Orbach requested a dialog with the HEP

community: "In making our plans for the

future, it is important to be conservative

and to learn from our experiences. Even

assuming a positive decision to build an

ILC, the schedules will almost certainly be

\section{Appendix A}

Completing the R\&D and engineering design, negotiating an international structure, selecting a site, obtaining firm financial commitments, and building the machine could take us well into the mid-2020s, if not later. Within this context, I would like to re-engage HEPAP in discussion of the future of particle physics. If the ILC were not to turn on until the middle or end of the 2020s, what are the right investment choices to ensure the vitality and continuity of the field during the next two to three decades and to maximize the potential for major discovery during that period?"

With the encouragement of the Office of Science and the support of Professor Mel Shochet, the chair of HEPAP, Fermilab will develop a strategic roadmap for the evolution of the accelerator-based HEP program, focusing on facilities at Fermilab that will provide discovery opportunities in the next two to three decades. This roadmap should keep the construction of the ILC as a goal of paramount importance. To guide this proposal, the Fermilab director has appointed a Steering Group consisting of members from Fermilab and the national particle and accelerator physics community to insure that the plan serves national needs. The Steering Group will also engage additional constituents in the analysis of the various physics opportunities.

The Steering Group will build the roadmap based on the recommendations of the EPP2010 National Academy of Sciences report and the recommendations of the P5 subpanel of HEPAP. The Steering Group should consider the Fermilab-based facilities in the context of the global particle physics program. Specifically the group should develop a strategic roadmap that:

1. supports the international $R \& D$ and engineering design for as early a start of the ILC as possible and supports the development of Fermilab as a potential host site for the ILC;

2. develops options for an accelerator-based high-energy physics program in the event the start of the ILC construction is slower than the technically-limited schedule; and

3. includes the steps necessary to explore higher energy colliders that might follow the ILC or be needed should the results from LHC point toward a higher energy than that planned for the ILC.

I am asking Deputy Director Kim to chair the Steering Group. Any recommendations that might be relevant to the FY09 budget should be transmitted as early as possible. The Steering Group's final report should be finished and delivered to the Fermilab director by August 1, 2007. This deadline would allow for presentations to the DOE and its advisory bodies before the structuring of the FY2010 budget. 


\section{Fermilab and the ILC}

\section{Accelerator}

Fermilab's International Linear Collider and superconducting radio-frequency program is coordinated with the ILC Global Design Effort and respects U.S. regional priorities. Fermilab's ILC effort focuses on the main linac, based on SCRF technology, and the design of conventional facilities, the largest cost drivers of the ILC. Key elements of Fermilab's main linac program include cavity and cryomodule fabrication and testing, related infrastructure development, advancing U.S. industrial capabilities, and developing designs and technologies to improve ILC performance and reduce cost. A collaboration of U.S. institutions under the leadership of the American Regional Team of the GDE is carrying out the U.S. ILC R\&D program, which will build and install SCRF infrastructure at U.S. laboratories including Fermilab. DOE has supported Fermilab to develop its SCRF infrastructure. The goal is to advance the ILC and to establish the U.S. and Fermilab as a credible, qualified host of the ILC. The technical goals are:

- Develop cavity-processing parameters for a reproducible cavity gradient of 35 $\mathrm{MV} / \mathrm{m}$; improve the yield of nine-cell cavities at $35 \mathrm{MV} / \mathrm{m}$ in vertical tests. Carry out parallel and coupled R\&D on cavity material, fabrication and processing to identify paths to success.

- Assemble and test several cryomodules with average gradient $>31.5 \mathrm{MV} / \mathrm{m}$.

- Build and test one or more ILC RF units at ILC beam parameters, high gradient and full pulse rep rate. Prepare the plans for and participate in the ILC main linac system test consisting of several RF units.

- Prepare infrastructure and test facilities to support continued development of cryomodules and to qualify industrially built main linac components and industrially built cryomodules.

The U.S. collaboration has fabricated and treated SCRF cavities for various projects at national laboratories. But the $35 \mathrm{MV} / \mathrm{m}$ technique of electropolishing is just starting. The U.S. ILC effort is expanding cavity-fabrication capability in industry and installing cavity-processing facilities to fulfill the needs of ILC R\&D. The goal for ILC cavities is 95 percent yield at $35 \mathrm{MV} / \mathrm{m}$. The U.S. goal is to fabricate, process and vertically test about 100 cavities per year, supporting the development of U.S. industrial capability. Thomas Jefferson National Accelerator Facility and Cornell University currently provide modest cavity-processing and testing capacity. New process and test infrastructure under construction at Argonne National Laboratory and Fermilab should allow the U.S. to meet its goal by 2009 , allowing the ILC to settle on a process and yield in about two years.

To complete the range of capabilities necessary for establishing core ILC technology in the U.S., Fermilab is installing an infrastructure to test dressed cavities with high-power RF, a cryomodule fabrication facility, and an RF unit test facility to examine cryomodules with an ILC-like beam.

Fermilab leads the effort to design a cryomodule for the ILC. Current efforts include moving the quadrupole to the center of the cryomodule to reduce vibration; developing cryogenic pipe sizes to support higher-gradient cavities; and designing longer cryogenic strings, symmetric cavity end-groups and a new tuner. Fermilab plans to build three cryomodules by the end of FY2010, assemble them into a single RF unit and test them. While this is an important milestone, U.S. preparation to build the ILC requires building tens of cryomodules in the U.S. and developing the industrial capability to produce hundreds.

In the engineering design phase of the ILC, Fermilab has committed to provide key engineers and scientists to develop the design of the ILC. Fermilab also plans to work with U.S. industry to improve cavity and cryomodule design. Accelerator physics design and simulation of the machine will focus on emittance preservation. While working with the worldwide ILC collaboration 
on the ILC machine design and global site development, Fermilab has special responsibilities to develop a Fermilab site-specific design for ILC.

\section{Physics and detectors}

Fermilab's ILC detector R\&D program supports the priorities established by Worldwide Study of the Physics and Detectors for Future Linear $e^{+} e^{-}$Colliders (http://physics.uoregon.edu/-lc/wwstudy/). Focusing on the most demanding aspects for the ILC detectors in collaboration with other laboratories and universities, the program's three areas of detector design are well matched to Fermilab's capabilities. This research is intended to have a broad approach, not limited to a single ILC detector concept.

The main focus is on silicon detectors, deployed either as pixel detectors or tracking detectors. The growing demands on detectors for ILC experiments require novel solutions of semiconductor detectors characterized by improvements in granularity, readout speed, radiation hardness, power consumption and sensor thickness. A current trend in the field of highly segmented ionizing radiation detectors is the development of monolithic active pixel sensors, which combine a pixel detector and readout electronics. Fermilab developers are vigorously pursuing vertical integrated systems with through-silicon via technology in a silicon-on-insulator process. This technology, whose development is driven by industry, holds enormous promise for providing low-mass, low-power particle physics detectors. An integrated approach studies the sensor technology and the mechanical design of vertex detectors as well as tracking detectors. The primary goal is to establish the proof of principle of each technology on a timescale compatible with the start of construction of the accelerator.

A second emphasis is on the characterization of pixelated photon detectors, a new development for photon detection. These PPDs consist of a pixelated silicon substrate, where each pixel operates as an avalanche photodiode in Geiger mode. These devices hold the promise of replacing the photomultiplier tubes. The devices are fast, operate at room temperature at modest bias voltages, and are insensitive to magnetic fields. Fermilab is working in close collaboration with universities on the characterization of these devices and on their applicability as photon detectors for use in dual-readout calorimeters and scintillator-based muon detection systems.

A third focal point is the development of a test-beam infrastructure. The ILC detectors are precision instruments using technologies never before employed in large-scale systems. Test beams will constitute a critical step in establishing the ILC detector technologies. In 2006, Fermilab upgraded its test-beam facility largely to satisfy the needs of the ILC. As a candidate host laboratory for the ILC and with limited availability of test beams at other laboratories over the course of the next few years, Fermilab intends to enhance testbeam facilities to accommodate the needs of the whole user community.

All detector R\&D builds on Fermilab's infrastructure and expertise. As a candidate host laboratory, Fermilab intends to increase the laboratory's effort in ILC-related activities including collaborative work on detector R\&D and test-beam facilities and strengthening its role in supporting users. The laboratory will foster a lively and diversified program of $\mathrm{R} \& \mathrm{D}$ projects, for their significance for crucial and cuttingedge technology developments related not just to the ILC but also to the principal themes of world-wide research in particle and astroparticle physics. The laboratory will foster synergies among projects to optimize the scientific output for an intense, costeffective, goal-oriented research program in collaboration with universities and other laboratories. Fermilab will continue to make the compelling case for ILC physics and to communicate with many audiences to strengthen the laboratory's leadership role in the ILC enterprise. 
The international neutrino program

While Europe and Japan have acceleratorbased programs in neutrino science, this section discusses the Japanese neutrino program.

The Japanese experiment $\mathrm{T} 2 \mathrm{~K}$ will be competitive with NOvA with respect to

\section{Appendix C}

NOvA is the only near-future experiment with possible sensitivity to the ordering of the states in the neutrino mass spectrum (the mass hierarchy).

Japan is considering a possible post-T2K program that would involve a $4 \mathrm{MW}$ beam from J-PARC sending neutrinos and antineutrinos to a new megaton-scale detector near the present Super-Kamiokande detector. Japanese and Korean researchers are giving some thought to the possibility of splitting the large new detector into two parts, one of which would be placed in Korea, approximately $1000 \mathrm{~km}$ from the J-PARC neutrino source. The figures on page 37 show the ability of this program to determine the mass hierarchy, and to establish the presence of $\mathrm{CP}$ violation, after four years of running with neutrinos, and another four years with antineutrinos. The curves, from M. Ishitsuka, T. Kajita, H. Minakata, and H. Nunokawa, Phys. Rev. D72, 033003 (2005), assume that the actual mass ordering is normal; curves for inverted ordering are similar.

By virtue of its longer baseline, the Project-X program would have better sensitivity to the neutrino mass ordering than the prospective J-PARC program, even if part of its megaton-scale detector is placed in Korea and much better sensitivity to this ordering if there is no detector in Korea. The figures on page 37 compare the potential sensitivities of the J-PARC and Project-X programs. The sensitivity to $\mathrm{CP}$ violation would be comparable in the U.S. and Japanese programs. The figures for the Project-X program use assumptions identical to those in the NuSAG report, except for the beam power. Water-Cerenkov (300 kt) and liquid-argon (100 kt) detector technologies would achieve similar sentitivities.
Quite apart from their relative sensitivities, the Japanese and U.S. programs, when combined, would be much stronger than either one alone, because they would operate under different physical conditions. In the U.S. program, there could be a wide-energy-band beam directed at a single large detector, possibly using liquid-argon technology, $1300 \mathrm{~km}$ away. In the Japanese program, there would be a much lower-energy, and narrower-band beam directed at either a single large water-Cerenkov detector $300 \mathrm{~km}$ away, or possibly a split version of this detector, with part of it $300 \mathrm{~km}$ from the neutrino source and the rest in Korea, about $1000 \mathrm{~km}$ from the source. Thanks to these differences between the U.S. and Japanese programs, together they would provide a much better probe of the mysteries of the neutrino world than either one alone. 


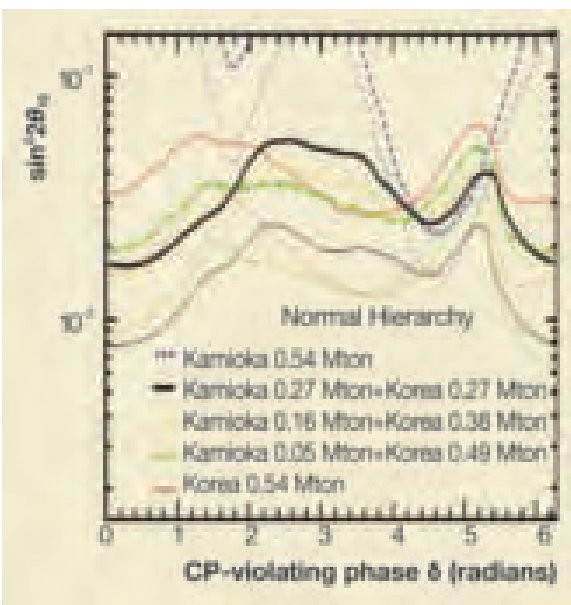

$2 \sigma$ (thin lines) and $3 \sigma$ (thick lines) sensitivity to the mass ordering by J-PARC upgrades and new detectors.

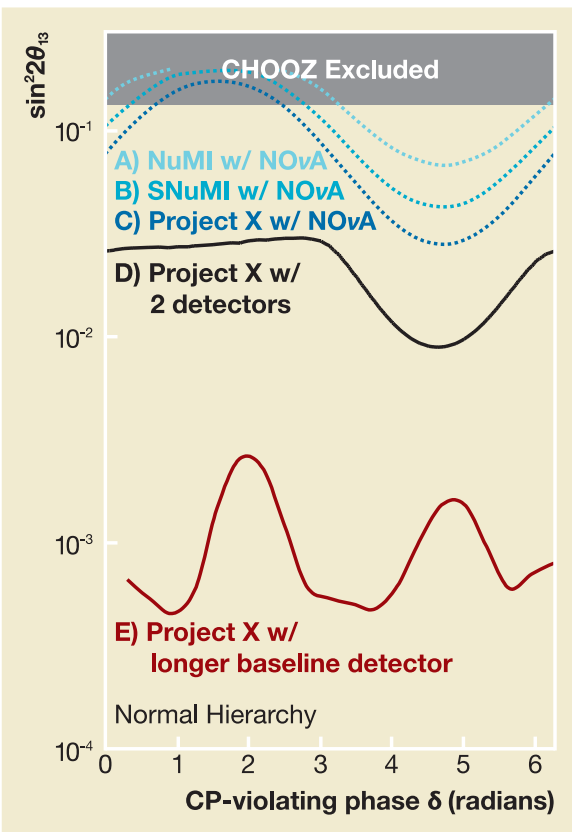

95 percent C.L. (dotted lines) and $3 \sigma$ (solid lines) sensitivity to the mass ordering assuming the normal mass hierarchy. For the inverted hierachy, flip each curve around a vertical line through $\delta=\pi$. All sensitivies assume 3 years of neutrino and 3 years of antineutrino running, corresponding to $15 \times 10^{20}, 30 \times 10^{20}, 60 \times 10^{20}$, and $120 \times 10^{20}$ protons on target for each neutrino type for NuMI, SNuMI, Project $X$ at $120 \mathrm{GeV}$, and Project $X$ at $60 \mathrm{GeV}$, respectively. Curves A-D use $120 \mathrm{GeV}$ protons and $\mathrm{E}$ uses $60 \mathrm{GeV}$ protons.

A) NOvA 15 kt detector with NuMI

B) NOvA 15 kt detector with SNuMI

C) NOvA 15 kt detector with Project $X$

D) Two $100 \mathrm{kt}$ LAr detectors at first $(\mathbf{7 0 0} \mathrm{km})$ and second $(810 \mathrm{~km})$ oscillation maxima using Project $X$ and the NuMI beamline.

E) One $100 \mathrm{kt} \operatorname{LAr}$ (equivalent to $\sim \mathbf{3 0 0} \mathrm{kt}$ water Cerenkov) detector at $\mathbf{1 3 0 0 ~} \mathbf{k m}$ using a wide-band neutrino beam with Project $X$.

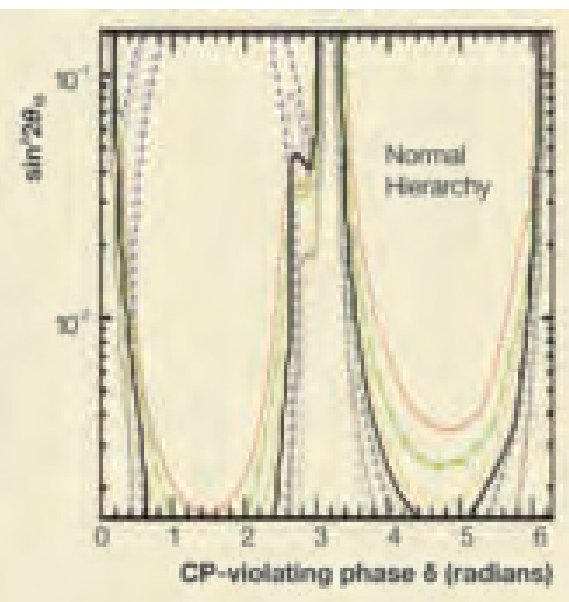

$2 \sigma$ (thin lines) and $3 \sigma$ (thick lines) sensitivity to CP violation by J-PARC upgrades and new detectors.

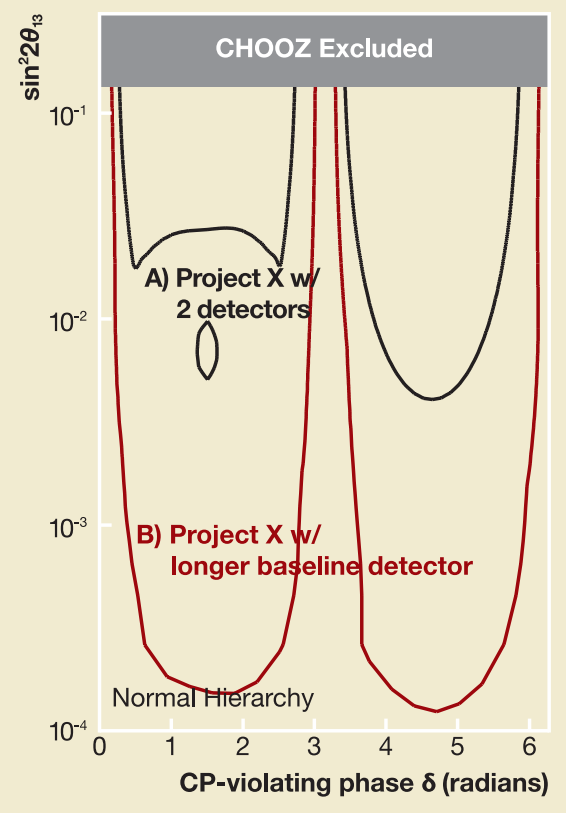

$3 \sigma$ sensitivity to $\mathrm{CP}$-violation assuming the normal mass hierarchy. For the inverted hierachy, flip each curve around a vertical line through $\delta=\pi$. All sensitivies assume 3 years of neutrino and 3 years of antineutrino running, corresponding to $60 \times 10^{20}$ and $120 \times 10^{20}$ protons on target for each neutrino type for Project $X$ at $120 \mathrm{GeV}$ and $60 \mathrm{GeV}$, respectively.

A) Two $100 \mathrm{kt}$ LAr detectors at first (700 km) and second $(810 \mathrm{~km})$ oscillation maxima using Project $X$ at $120 \mathrm{GeV}$ and the NuMI beamline.

B) One $100 \mathrm{kt}$ LAr (equivalent to $\mathbf{3 0 0} \mathrm{kt}$ water Cerenkov) detector at 1300 km using a wide-band neutrino beam with Project $X$ at $60 \mathrm{GeV}$. 


\section{Neutrino science with $8 \mathrm{GeV}$ and $800 \mathrm{GeV}$ protons}

This section lists some experiments with neutrino beams that could be carried out at proton facilities. Possible long-baseline programs for neutrino oscillation and $\mathrm{CP}$ violation are not discussed here.

\section{Appendix D}

\section{Neutrino-science experiments with $8 \mathrm{GeV}$ protons}

The excess of low-energy electron-neutrino-

like events recently observed by MiniBooNE could arise either from new physics, not compatible with simple two-flavor oscillations, or from a new kind of background that is of importance for oscillation experiments operating in this energy range. An experiment dubbed microBooNE with excellent lowenergy sensitivity provided by a liquid argon time projection chamber is proposed to study individual final states producing events in the region of excess. This experiment would also be an extremely valuable step in demonstrating the effectiveness of LArTPCs for sensitive discrimination of backgrounds to neutrino interactions. If the experiment is sited in the MINOS surface building, it would be exposed to the Booster neutrino beam to accomplish microBooNE. It would also be exposed to a far off-axis NuMI beam, providing useful study of low-energy neutrinos, although it may be desirable to have a LAr detector down in the NuMI tunnel to act as a NOvA near detector. Both detector sites would produce useful neutrino scattering measurements relevant for oscillation physics, as well as scattering measurements of relevance for nuclear physics. Smaller scale LAr experiments like this one can provide very useful experience toward potential long-baseline detectors.

The strange quark contribution to nucleon spin $(\Delta s)$ can be extracted from neutral current elastic scattering in the Booster neutrino beam with higher precision and less model dependence than in deep-inelastic scattering measurements. In addition to providing the strange quark piece of the proton spin puzzle, the $\Delta s$ measurement has cosmological implications, as NC-elastic interactions dominate in core-collapse supernovae. At present, $\Delta s$ results from polarized, inclusive, lepton deepinelastic scattering and from semi-inclusive leptonic deep-inelastic scattering are not consistent with each other. Although, given additional run time beyond that currently approved, the SciBooNE experiment could better measure the ratio of $\mathrm{NC}$-elastic scattering to charged-current scattering events, a fully sensitive experiment might require detector upgrades to SciBooNE. Required sensitivity is currently being studied.

Neutrino-nucleus cross-sections in the low energy (tens of $\mathrm{MeV}$ ) regime for a number of nuclear targets pertinent to the process of supernova core collapse can be studied using a neutrino beam generated from stopped pions produced by very intense proton beams of $1-2 \mathrm{GeV}$, and an experiment similar to NuSNS at the Spallation Neutron Source. In addition, coherent elastic scattering of neutrinos off nuclear targets could possibly be measured, providing a precision test of the Standard Model not possible at the SNS because of neutron backgrounds.

\section{Neutrino-science experiments with $800 \mathrm{GeV}$ protons}

Exciting experiments using high-energy neutrinos produced in a Tevatron fixed-target neutrino beam line could be performed if sufficient $120 \mathrm{GeV}$ protons from the Main Injector are available to feed both the long-baseline neutrino program and the Tevatron. For example, a precision measurement of the weak mixing angle $\theta_{\mathrm{W}}$ using muon-neutrino scattering on electrons performed with a high-energy neutrino beam could probe Beyond the Standard Model physics in a way complementary to other electroweak measurements. Tension that presently exists in global electroweak fits perhaps hints at beyond Standard Model effects. Only measurements of the invisible width of the $Z$ in electron-positron collisions probe the Standard Model in the same way. Such a measurement of $\theta_{\mathrm{W}}$ could be performed by an experiment dubbed $\mathrm{NuSOnG}$ that would utilize a new spectrometer in a pure muon-neutrino or muon-antineutrino beam generated by 
$800 \mathrm{GeV}$ protons from the Tevatron with a sign-selected quadrupole train. A measurement of $\sin ^{2} \theta_{\mathrm{W}}$ in the scattering of neutrinos off electrons to 0.7 percent could be produced with $2 \times 10^{20}$ protons on target. Such an experiment could not be performed by any other neutrino beam at Fermilab, CERN or J-PARC.

Upgrade to the Fermilab proton facility During the era of NOvA operations, neutrino experiments in Booster or Tevatron neutrino lines cannot be supported without compromising NOvA physics, unless upgrades are made to the Fermilab proton accelerator complex. The SNuMI upgrade would increase the sensitivity and physics reach of the NOvA program. It would also increase the competitiveness of NOvA with its contemporary neutrino-oscillation experiments. However, SNuMI could not simultaneously provide adequate $8 \mathrm{GeV}$ beam power for experiments such as microBooNE or SciBooNE with upgrades. A precision electroweak neutrino experiment, such as $\mathrm{NuSOnG}$, would require about 5 percent of the SNuMI $120 \mathrm{GeV}$ beam power. Project $\mathrm{X}$, on the other hand, would provide ample proton beam power to provide both a greater than three-fold increase in $120 \mathrm{GeV}$ beam power for NOvA and future long-baseline experiments and more than ample $8 \mathrm{GeV}$ beam power for neutrino experiments. The $120 \mathrm{GeV}$ beam power available with Project $\mathrm{X}$ would also allow operation of a Tevatron fixed-target neutrino line without noticeable impact on the long-baseline neutrino program. Thus, Project $\mathrm{X}$ would enable a program of neutrino experiments that would not otherwise be feasible, while greatly enhancing the physics reach of long-baseline neutrinooscillation experiments. 


\section{Appendix E}

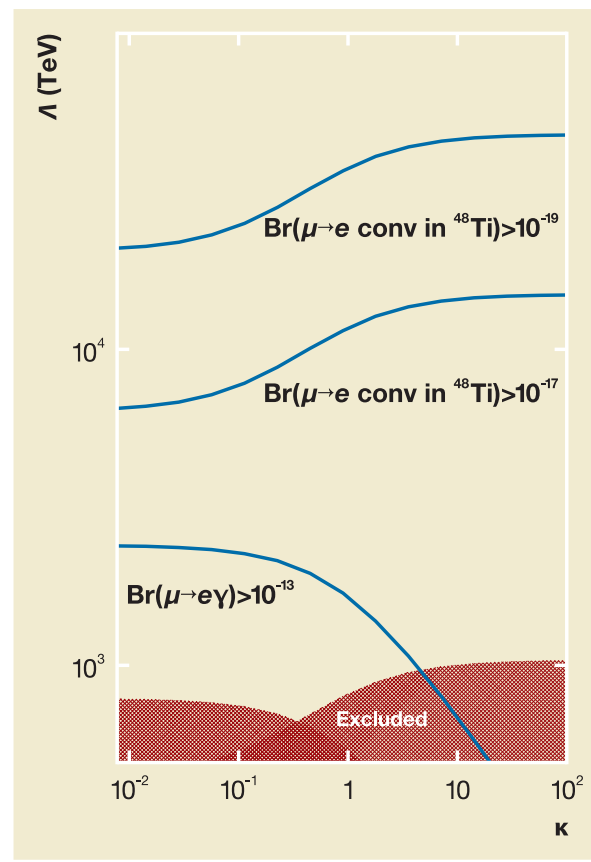

The sensitivity to a new physics scale $\Lambda$ in a $\mu \rightarrow \boldsymbol{e}$ conversion experiment in ${ }^{48} \mathrm{Ti}$ that can probe a normalized capture rate of $10^{-17}$ and $10^{-19}$, and a $\mu \rightarrow$ ey search that is sensitive to a branching ratio of $10^{-13}$. The sensitivity is plotted as a function of $\mathbf{K}$, a parameter that interpolates between a flavor transition magnetic moment-type operator $(K<<1)$ and a lepton-flavor changing four-fermion operator $(K>>1)$. Also depicted is the region already excluded from searches for both $\mu \rightarrow e \gamma$ and $\mu \rightarrow e$ conversion in ${ }^{48} \mathrm{Ti}$.

\section{Muon-to-electron conversion in nuclei}

With the discovery of neutrino masses and mixings we have learned that neutral lepton flavor quantum numbers are violated in nature. An immediate question arises: "Does lepton flavor violation also occur at an appreciable rate with the charged leptons?" While the Standard Model predicts negligible rates for charged lepton flavor violation, many models, including various versions of supersymmetry, predict CLFV at an appreciable and potentially observable rate. Searches for CLFV are the most powerful and promising probes for new physics at and above the $\mathrm{TeV}$ scale.

Rare muon processes provide the deepest CLFV probes due to the copious production of muons in proton fixedtarget collisions. SNuMI and Project X are capable of extending the current sensitivity by many orders of magnitude. Current experiments have been able to rule out, at the 90 percent confidence level, $\mu \rightarrow e \gamma$ with branching ratios above $1.1 \times 10^{-11}, \mu \rightarrow e e e$ with branching ratios above $1.0 \times 10^{-12}$, while the rate for $\mu+{ }^{48} \mathrm{Ti} \rightarrow e+{ }^{48} \mathrm{Ti}$ normalized to the capture rate ( $\mu \rightarrow e$ conversion in titanium) is constrained to be less than $4.3 \times 10^{-12}$. Various scenarios for new physics at the $\mathrm{TeV}$ scale predict these processes to occur with rates that are close to these current bounds. In fact, searches for CLFV in muon processes already provide the most stringent constraints on some new physics scenarios. The bounds on many scenarios became even more stringent with the discovery that neutrinos have nonzero masses and large mixing angles.

One can estimate the new physics expectations for the rates for different muon CLFV processes in a model independent way. For concreteness, consider the effect of adding to the Standard Model the following CLFV effective Lagrangian:
Here $\Lambda$ sets the scale of new physics and $\kappa$ interpolates between a pure transition magnetic-moment-type operator $(\kappa<<1)$ and a pure four-fermion interaction $(\kappa>>1)$. This effective Lagrangian describes both $\mu \rightarrow e \gamma$ and $\mu \rightarrow e$ conversion (and, at a less significant level, $\mu \rightarrow e e e$, which will not be discussed). It qualitatively captures the predictions of most new physics scenarios containing CLFV. The potential experimental sensitivity to $\Lambda$ and $\kappa$ is depicted in the figure. Overall, an experiment sensitive to $\mu \rightarrow e$ conversion rates larger than $10^{-17}$ is probing a fundamental new physics scale $\Lambda$ up to several thousands of $\mathrm{TeV}$, regardless of the value of $\kappa$.

For $\kappa<<1$, the normalized $\mu \rightarrow e$ conversion rate is at least several times $10^{-3}$ times the branching ratio for $\mu \rightarrow e \gamma$, while for $\kappa>>1$ the branching ratio for $\mu \rightarrow e \gamma$ is many orders of magnitude smaller than the normalized capture rate for $\mu \rightarrow e$ conversion. A $\mu \rightarrow e$ conversion experiment at the $10^{-17}$ level is at least as sensitive to new physics as a $\mu \rightarrow e \gamma$ experiment at the $10^{-14}$ level. Hence, a $\mu \rightarrow e$ conversion experiment associated with Project X could probe CLFV physics beyond the reach of the Muon-to-Electron-Gamma experiment, which will start taking data at the Paul Scherrer Institute soon and aims at being sensitive to $\mu \rightarrow e \gamma$ if its branching ratio is above $10^{-13}$.

In the case of a confirmed signal, combined results from different CLFV searches would provide detailed information regarding the underlying new physics. In particular a versatile $\mu \rightarrow e$ conversion experimental set-up has the unique capability of being able to distinguish the underlying effective operators responsible for CLFV (in the example above, this means measuring $\kappa$ as well as $\Lambda$ ) by observing the conversion rate on different nuclear targets. 
Precision physics experiments with kaon beams

The charged kaon experiment enabled by the Tevatron Stretcher and Project X could deliver a precision measurement of the $K^{+} \rightarrow \pi^{+} v \bar{v}$ branching fraction that matches the small theoretical uncertainty. In parallel, the charged kaon experiment

\section{Appendix F} including a precision measurement of $K^{+} \rightarrow e^{+} v$ and a search for $K^{+} \rightarrow \pi^{+} \mu e$ which are both uniquely incisive probes of Terascale physics and beyond. The neutral kaon experiment driven by the enormous $8 \mathrm{GeV}$ beam power provided by Project $\mathrm{X}$ could discover and measure the ultrarare $K_{L} \rightarrow \pi^{0} v \bar{v}$ process. As the sensitivity reaches down to the Standard Model branching ratio of $3 \times 10^{-11}$, several extensions of the Standard Model would be excluded or, even better, new Terascale phenomena would be detected. Upon attaining the Standard Model sensitivity the neutral kaon experiment then becomes sensitive to very high mass scale $\left(>1000 \mathrm{TeV} / \mathrm{c}^{2}\right)$ through precision measurement of the $K_{L} \rightarrow \pi^{0} v \bar{v}$ branching fraction.

Driving the charged kaon experiment with the high-duty-factor Tevatron Stretcher simultaneously reduces detector rates by a factor of three and reduces the proton tax on the Main Injector neutrino program from 30 percent to five percent. The lower detector rates reduce the technical risk of the charged kaon experiment and support scaling of the experiment to much higher sensitivities in the Project-X era. The high-energy separated charged-kaon beam based on ILC crab-cavity technology drives this next step in ultrarare $K^{+} \rightarrow \pi^{+} v \bar{v}$ sensitivity with samples of 100-200 decays per year within reach prior to Project X coming online. Project X could then further increase the rare-decay sensitivities by a factor of three while maintaining a small five percent tax on the Main Injector neutrino program. Meanwhile at CERN the NA48 collaboration is working to advance a $K^{+} \rightarrow \pi^{+} v \bar{v}$ experiment that could collect about 50 events per year early in the next decade. The key technical elements of the charged-kaon experiment

have been reviewed worldwide in detail, and an experiment could be developed into a reviewable project with a year of effort.

Estimates of kaon flux driven from 8 $\mathrm{GeV}$ Fermilab proton sources suggest that a compelling staged neutral-kaon program could be developed that first reaches Standard Model sensitivity of the $K_{L} \rightarrow \pi^{0} v \bar{v}$ process with an experiment driven by the Booster, followed by a precision measurement enabled by the $8 \mathrm{GeV}$ proton flux provided by Project X. Across the globe a similar staged $K_{L} \rightarrow \pi^{0} v \bar{v}$ program is being pursued in Japan, where Stage I at KEK is now complete. There are plans for Stage II at J-PARC which could reach Standard Model sensitivity sometime in the next decade. Key technical issues of the neutral kaon experiment are actively being pursued worldwide, and an experiment based at Fermilab could be developed into a reviewable project with a year of effort. 


\section{Facilities considered}

The Steering Group considered 12 facilities.

The table below lists the facilities that were not described in Section 4.

\section{Appendix G}

\begin{tabular}{|c|c|c|c|c|}
\hline Facility & Description & Performance Parameters & Physics Program & ILC Synergy \\
\hline \multicolumn{5}{|l|}{ Proton Facilities } \\
\hline $\begin{array}{l}\text { LHC Luminosity } \\
\text { Upgrade }\end{array}$ & $\begin{array}{l}\text { Luminosity upgrade } \\
\text { based on high } \\
\text { performance IR } \\
\text { quadrupoles based on } \\
\mathrm{Nb}_{3} \mathrm{Sn} \text { technology. }\end{array}$ & $\mathrm{L}>1 \times 10^{35} \mathrm{~cm}^{-2} \mathrm{~s}^{-1}$ & Energy frontier & No \\
\hline $\begin{array}{l}\text { Proton Complex } \\
\text { Upgrade }\end{array}$ & $\begin{array}{l}\text { New } 8 \mathrm{GeV} \text { Booster } \\
\text { fed by a new } 1 \mathrm{GeV} \\
\text { linac }\end{array}$ & $\begin{array}{l}2.3 \mathrm{MW} \text { beam power } \\
\text { at } 120 \mathrm{GeV}\left(23 \times 10^{20}\right. \\
\text { protons/year) } \\
8 \mathrm{GeV} \text { slow spill available } \\
\text { by diverting protons from } \\
\text { the } 120 \mathrm{GeV} \text { program }\end{array}$ & $\begin{array}{l}\text { Neutrino science } \\
\text { and precision } \\
\text { physics }\end{array}$ & No \\
\hline Antiproton Facility & $\begin{array}{l}\text { Continued opera- } \\
\text { tion of the } \\
\text { Antiproton Source }\end{array}$ & $\begin{array}{l}2 \times 10^{11} \text { protons/hour at } \\
8 \mathrm{GeV} \text {. Operated in storage } \\
\text { mode. } \\
\text { Incompatible with SNuMI. } \\
\text { Minor hit on proton } \\
\text { availability from Project X. }\end{array}$ & Precision physics & No \\
\hline $\begin{array}{l}\text { High Energy, } \\
\text { High Power } \\
v \text { Beam }\end{array}$ & $\begin{array}{l}480 \mathrm{GeV} \text { dual } \\
\text { aperture accelerator } \\
\text { constructed in the } \\
\text { Tevatron tunnel. } \\
\text { Based on superferic } \\
\text { magnet. }\end{array}$ & $\begin{array}{l}\sim 5 \mathrm{MW} \text { beam power } \\
\text { at } 480 \mathrm{GeV}\left(25 \times 10^{20}\right. \\
\text { protons/year) }\end{array}$ & Neutrino science & No \\
\hline
\end{tabular}

\section{Electron Facilities}

\begin{tabular}{|c|c|c|c|c|}
\hline $6 \mathrm{GeV}$ ILC Linac & $\begin{array}{l}\text { ILC } 1 \% \text { systems test } \\
\text { in ILC-like tunnel }\end{array}$ & $\begin{array}{l}\text { ILC beam parameters } \\
9 \mathrm{ma} \times 1 \mathrm{~ms} \times 5 \mathrm{~Hz}\end{array}$ & NA & Yes \\
\hline Giga-Z & $\begin{array}{l}90 \mathrm{GeV} \text { linear collider } \\
\text { based on ILC } \\
\text { technology }\end{array}$ & $\begin{array}{l}10^{9} \mathrm{Z} \text { 's } \\
\mathrm{L}>1 \times 10^{33} \mathrm{~cm}^{-2} \mathrm{~s}^{-1}\end{array}$ & Precision physics & Yes \\
\hline Super B Factory * & $\begin{array}{l}\text { Asymmetric }(4 \mathrm{GeV} \times 7 \\
\mathrm{GeV}) \mathrm{e}^{+} e^{-} \text {collider } \\
\text { in the Tevatron tunnel }\end{array}$ & $\mathrm{L}>1 \times 10^{36} \mathrm{~cm}^{-2} \mathrm{~s}^{-1}$ & Precision physics & $\begin{array}{l}\text { No, unless } \\
\text { converted ILC } \\
\text { damping ring }\end{array}$ \\
\hline $\begin{array}{l}\text { ILC Damping } \\
\text { Ring }\end{array}$ & $\begin{array}{l}5 \text { GeV ILC damping } \\
\text { ring in the Tevatron } \\
\text { tunnel. }\end{array}$ & $\begin{array}{l}\text { ILC damping ring } \\
\text { parameters }\end{array}$ & $\begin{array}{l}\mathrm{NA} \text {, unless } \\
\text { converted to use } \\
\text { as } B \text { Factory }\end{array}$ & Yes \\
\hline
\end{tabular}

* $B$ physics with Super B Factory: A second generation $B$ Factory with luminosity above $10^{36} \mathrm{~cm}^{-2} \mathrm{~s}^{-1}$ providing data samples of 50-100 $\mathrm{ab}^{-1}$ can explore a wide range of physics beyond the Standard Model. In many scenarios, the physics reach extends beyond the TeV scale and the pattern of deviation from Standard Model predictions can help distinguish between models. The possibility of constructing a Super B Factory at Fermilab should be re-examined sometime around 2012 in light of LHC discoveries, progress on ILC development, and worldwide plans for Super B factories elsewhere. 
Steps toward a muon collider

This section lists the steps required to demonstrate the viability of a muon collider, and current activities.

\section{End-to-end conceptual design}

The "front end" of a muon collider and that of a neutrino factory have much in

\section{Appendix H}

has shown, it is useful to develop an end-to-end design to illuminate the further simulation, design and hardware R\&D needed for development of a facility; to derive early cost estimates; and to evaluate viability. Such exercises have been carried out.

\section{Proton driver}

To achieve luminosities of $\mathrm{O}\left(10^{34} \mathrm{~cm}^{-2} \mathrm{~s}^{-1}\right)$ requires proton power on target of about 4 MW in the form of approximately 3 ns-long bunches each with of $\mathrm{O}\left(10^{14}\right)$ protons. This driver would be an upgrade of Project X. Some accumulator, from an appropriate source, with fast extraction would need development.

\section{Targeting, capture and phase rotation}

While several multimegawatt target developments have been carried out, each has special features, and the muon collider target is no exception. An international experiment is now underway using a mercury jet and the requisite peak proton intensity. Other target schemes need further investigation. Capture and phase rotation require very-high-field solenoids and low-frequency cavities or induction accelerator units that can operate in magnetic fields, all of which need R\&D.

\section{D ionization cooling}

Ionization cooling is a key process for both the neutrino factory and muon collider.

The neutrino factory requires only transverse cooling (4D) by about a factor of 100 in the phase space area to produce a useful neutrino beam. A muon collider, however, requires a $6 \mathrm{D}$ phase space volume reduction of $10^{6}$. So far, neither has been demonstrated, although a $4 \mathrm{D}$ cooling experiment is now about three years from data taking. Current ideas envision three different configurations for performing the $6 \mathrm{D}$ cooling, but no complete experiment testing any of them is yet designed. All schemes use high magnetic fields and high-gradient cavities, preferably immersed in high-magnetic fields together with energy loss-cells either separate or incorporated into the reaccelerating cavities. All of these items require performance well beyond the current state of the art.

\section{Reacceleration}

After cooling, the muons must be rapidly accelerated to the full collision energy. Schemes using linacs, recirculating linacs, fixed-field alternating-gradient accelerators, pulsed synchrotrons and combinations of these have been suggested. High-gradient, relatively low-frequency superconducting cavities and other accelerator technology beyond today's practice require design and development in an iterative cycle with system design to understand the optimum approach and cost for a given target luminosity.

\section{Collider ring}

Maximizing the luminosity requires a very-high-magnetic-field storage ring formed of magnets with great radiation tolerance. Both conditions are far from current practice and would require a concerted design and development program for feasibility and economic assessment. The design of the focusing lattice is also very challenging in its demand for low-momentum compaction and high-momentum acceptance.

\section{Detector}

Besides the challenges of detection in a high-luminosity lepton environment, a muon collider detector must deal successfully with a very high radiation background caused by the muon decay electrons. This problem has received some consideration in the past, but the advances of detector technology require continuing reevaluation.

\section{Program elements}

In addition to the several technology R\&D matters that require resolution for an evaluation of muon collider viability, 
extensive simulation and design activities are required. Some technology $R \& D$ items are

- high-field magnets, including solenoids, dipoles and quadrupoles, with the development of accompanying superconducting materials, including high-temperature superconductor,

- high-gradient RF cavities, both normal and superconducting, of various frequencies with normal-conducting cavities immersed in magnetic fields,

- liquid or high pressure gaseous hydrogen or $\mathrm{LiH} \mathrm{dE/dx} \mathrm{cells,}$

- auxiliary technologies.

The simulation and design work that is required across the board is often neglected in evaluating needed resources.

\section{Current activities}

A worldwide collaboration currently looking at neutrino factories expects to issue a report in 2012 reviewing the physics as it appears then and presenting possibilities for discovery. Currently the international MERIT experiment at CERN is exploring the mercury jet production target at the needed peak power level. In the U.S., the Neutrino Factory and Muon Collider Collaboration of laboratory and university scientists, together with international partners, is performing the MUCOOL activities at Fermilab to develop muon cooling technologies. NFMCC is coordinating U.S. participation in the Muon Ionization Cooling Experiment at Rutherford Appleton Laboratory to carry out a 4D ionization cooling and demonstration project. In addition, Fermilab has commissioned a Muon Collider Task Force to explore long-term prospects of a muon collider. 


\section{Acknowledgement}

The Fermilab Steering Group is grateful to many members of the particle physics and accelerator community for their expert advice and contributions to the preparation of this report:

Robert Abrams, Nikolai Andreev, Charles Ankenbrandt, Giorgio Apollinari, Jeff Appel, Rick Baartman, Jonathan Bagger, Wander Baldini, Barry Barish, Gerald Blazey, Dixon Bogert, Ed Bonnema, James Brau, Steven Brice, Stanley Brodsky, Daniel Broemmelsiek, Alan Bross, Charles Brown, Douglas Bryman, William Bugg, Alexey Burov, Phillip Burrows, Maksim Bychkov, Robert Cahn, Marcela Carena, Jean-Paul Carneiro, Harry Carter, Robert Carey, Brian Chase, David Christian, Michael Church, Willie Clark, Janet Conrad, John Corlett, Mary Anne Cummings, Dan Dale, Chris Damerell, Paul Debevec, André de Gouvea, Fritz DeJongh, Marcel Demarteau, Paul Derwent, Milind Diwan, Harold Lee Dodds, Gerald Dugan, Craig Dukes, Estia Eichten, Eckhard Elsen, Louis Emery, Peter Fisher, George Flanagan, Tony Favale, Gary Feldman, Buck Field, David Finley, Bonnie Fleming, Brian Foster, Emil Frlez, Kenneth Ganezer, Consolato Gattuso, Stephen Geer, Norman Gelfand, Terry Goldman, Keith Gollwitzer, Maury Goodman, Paul Grannis, Daniel Green, Terry Grimm, Alan Hahn, Reidar Hahn, Michael Harrison, Ayman Hawari, David Hertzog, Robert Hirosky, David Hitlin, Richard Holmes, Maxine Hronek, Patrick Hurh, Jim Hylen, Gerald Jackson, Andreas Jansson, David Johnson, Jeffrey Johnson, Rol Johnson, John Johnstone, Chang Kee Jung, Steven Kahn, Peter Kammel, Yuri Kamyshkov, David Kawall, Kara Keeter, Christina Keller, Robert Kephart, Harold Kirk, David Kirkby, Arkadiy Klebaner, Sergey Korenev, Ioanis Kourbanis, Andreas Kronfeld, Krishna Kumar, James Lackey, Kenneth Lande, Valeri Lebedev, Kevin Lesko, Tony Leveling, Mats Lindroos, Laurence Littenberg, Vladimir Lobashev, Kevin Lynch, William Marciano, Daniel Marlow, John Marriner, Michael Martens, Dongming Mei, Mark Messier, Peter Meyers, Phillip Miller, Shekhar Mishra, Hugh Montgomery, Kevin Munday, Homer Neal, David Neuffer, Andrew Norman, Kenneth Olsen, Peter Ostroumov, Satoshi Ozaki, Robert Palmer, Vaia Papadimitriou, Stephen Parke, Kent Paschke, Ralph Pasquinelli, Todd Pedlar, Stephen Peggs, Susan Pfiffner, Henryk Piekarz, Thomas Phillips, Dinko Pocanic, Milorad Popovic, James Popp, Eric Prebys, Chris Quigg, Regina Rameika, Ronald Ray, Lee Roberts, Tom Roberts, Natalie Roe, Jerome Rosen, Marc Ross, Howard Rubin, Randy Ruchti, Richard Sah, Niki Saoulidou, Kate Scholberg, Alan Schwartz, Yannis Semertzidis, Abraham Seiden, Melvyn Shochet, Marilyn Smith, Henry Sobel, Paul Souder, Giulio Stancari, Michelle Stancari, Raymond Stefanski, James Stone, Sheldon Stone, Michael Syphers, Alex Tarasiewicz, Eddie Tatar, Rex Tayloe, Alvin Tollestrup, Yagmur Torun, Todd Treado, Michael Turner, Fred Ullrich, John Urbin, Alexander Valishev, Leonid Vorobiev, Nick Walker, Robert Webber, Bernard Wehring, Steven Werkema, Christopher White, Herman White, James Whitmore, David Wildman, Kent Alan Williams, William Willis, Phil Winkle, William Snow, Stanley Wojcicki, Hitoshi Yamamoto, Peter Yamin, Katsuya Yonehara, Cary Yoshikawa, Albert Young, Michael Zeller, Michael Zisman, Alexander Zlobin, and Robert Zwaska. 
Fermilab

Fermi National Accelerator Laboratory

Kirk and Wilson Streets

Batavia Illinois 60510-0500

6308403000

www.fnal.gov 